\title{
Computational neuroanatomy of human stratum proprium of interparietal sulcus
}

\author{
Maiko Uesaki $^{1,2,3} \cdot$ Hiromasa Takemura ${ }^{2,4,5} \cdot$ Hiroshi Ashida ${ }^{1}$
}

Received: 24 March 2017/ Accepted: 4 August 2017/Published online: 4 September 2017

(C) The Author(s) 2017. This article is an open access publication

\begin{abstract}
Recent advances in diffusion-weighted MRI (dMRI) and tractography have enabled identification of major long-range white matter tracts in the human brain. Yet, our understanding of shorter tracts, such as those within the parietal lobe, remains limited. Over a century ago, a tract connecting the superior and inferior parts of the parietal cortex was identified in a post-mortem study: stratum proprium of interparietal sulcus (SIPS; Sachs, Das hemisphärenmark des menschlichen grosshirns. Verlag von georg thieme, Leipzig, 1892). The tract has since been replicated in another fibre dissection study (Vergani et al., Cortex 56:145-156, 2014), however, it has not been fully investigated in the living human brain and its precise anatomical properties are yet to be described. We used dMRI and tractography to identify and characterise SIPS in vivo, and explored its spatial proximity to the cortical
\end{abstract}

Electronic supplementary material The online version of this article (doi:10.1007/s00429-017-1492-1) contains supplementary material, which is available to authorised users.

Maiko Uesaki

uesaki@gst.ritsumei.ac.jp

$\triangle$ Hiromasa Takemura

htakemur@nict.go.jp

1 Department of Psychology, Graduate School of Letters, Kyoto University, Kyoto, Japan

2 Japan Society for the Promotion of Science, Tokyo, Japan

3 Open Innovation and Collaboration Research Organization, Ritsumeikan University, Osaka, Japan

4 Center for Information and Neural Networks (CiNet), National Institute of Information and Communications Technology, and Osaka University, Suita, Japan

5 Graduate School of Frontier Biosciences, Osaka University, Suita, Japan areas associated with optic-flow processing using fMRI. SIPS was identified bilaterally in all subjects, and its anatomical position and trajectory are consistent with previous post-mortem studies. Subsequent evaluation of the tractography results using the linear fascicle evaluation and virtual lesion analysis yielded strong statistical evidence for SIPS. We also found that the SIPS endpoints are adjacent to the optic-flow selective areas. In sum, we show that SIPS is a short-range tract connecting the superior and inferior parts of the parietal cortex, wrapping around the intraparietal sulcus, and that it may be a crucial anatomy underlying optic-flow processing. In vivo identification and characterisation of SIPS will facilitate further research on SIPS in relation to cortical functions, their development, and diseases that affect them.

Keywords Stratum proprium of interparietal sulcus . Diffusion-weighted MRI · Tractography · fMRI · Optic flow $\cdot$ Visuo-vestibular integration

\section{Introduction}

Anatomical connections through the white matter axon bundles (i.e. fascicles, tracts) establish fundamental features of the brain's information processing (Catani and Ffytche 2005; Catani and Thiebaut de Schotten 2012; Bullock et al. 2005; Fields 2008, 2015; Wandell and Yeatman 2013; Wandell 2016). Diffusion-weighted magnetic resonance imaging (dMRI) and tractography provide a unique opportunity to identify and characterise the white matter tracts in the living human brain (Catani et al. 2002; Wakana et al. 2004; Mori and Zhang 2006; Catani and Thiebaut de Schotten 2012; Craddock et al. 2013; Wandell 2016; Rokem et al. 2017). 
A body of dMRI research has successfully identified several major long-range white matter tracts, such as the superior longitudinal fasciculus and the inferior longitudinal fasciculus, in a consistent manner with the known postmortem anatomy (Catani et al. 2002; Wakana et al. 2004; Schmahmann et al. 2007), and ergo opened new avenues to research on the properties of major human white matter tracts in relation to development and diseases (Lebel et al. 2012; Yeatman et al. 2012a, 2014a; Ogawa et al. 2014; Malania et al. 2017). More recent dMRI studies have identified shorter white matter tracts including frontal aslant tract and vertical occipital fasciculus, which, partially for their relatively short trajectories, had previously received very little attention in the neuroscience literature (Thiebaut de Schotten et al. 2012; Catani and Thiebaut de Schotten 2012; Catani et al. 2013; Yeatman et al. 2013, 2014b; Takemura et al. 2016b, 2017). Some of those studies have suggested the importance of the shorter tracts in relation to cognitive functions and diseases (Kinoshita et al. 2015; Kemerdere et al. 2016; Kronfeld-Duenias et al. 2016; Duan et al. 2015; Takemura et al. 2016b; Lee Masson et al. 2017).

Here, we focus on a short white matter tract connecting the superior and inferior parts of the parietal cortex, wrapping around the inferior parietal sulcus. This tract was initially described by the German neurologist Heinrich Sachs (1892) as the stratum proprium of interparietal sulcus (hereafter, we refer to this tract as SIPS). Except for one recent fibre dissection study replicating Sachs's work (Vergani et al. 2014), this tract has been largely overlooked in the literature. Given the functional MRI (fMRI) evidence indicating the involvement of the superior and inferior parts of the parietal cortex in crucial cognitive functions (Corbetta and Shulman 2002; Culham et al. 2006; Uncapher and Wagner 2009; Cardin and Smith 2010; Blanke 2012; Greenlee et al. 2016), SIPS is likely a necessary and important tract supporting those functions. Yet, the characteristics of SIPS are poorly understood due to the lack of studies replicating SIPS in the living human brain, using three-dimensional digital anatomical data such as dMRI and reproducible computational analyses.

One of the cortical functions that involve the parietal cortex is optic-flow processing. Optic flow is the pattern of visual motion signals elicited by self-motion (Gibson $1950,1954)$, and is an important cue to accurate perception of self-motion. A network of sensory areas in the parietal cortex has been shown to be involved in optic-flow processing (Cardin and Smith 2010, 2011). Those optic-flow selective areas include the ventral intraparietal area (VIP), the precuneus motion area $(\mathrm{PcM})$ and the putative area $2 \mathrm{~V}$ $(\mathrm{p} 2 \mathrm{v})$ located in the superior part of the parietal cortex, and the posterior-insular complex (PIC+: Deutschländer et al. 2004; Cardin and Smith 2010, 2011; Biagi et al. 2015; Uesaki and Ashida 2015; Wada et al. 2016) in the inferior part of the parietal cortex. Several studies have described a convergence of visual and vestibular information regarding self-motion, involving those optic-flow selective areas (Kleinschmidt et al. 2002; Wiest et al. 2004; Kovács et al. 2008; Fetsch et al. 2009; Butler et al. 2010; Prsa et al. 2015; Uesaki and Ashida 2015). To fully understand the underlying mechanism of optic-flow processing, it is essential to investigate how communication between the superior and inferior parts of the parietal cortex is supported by the white matter anatomy.

We used dMRI and tractography to identify the anatomical location and trajectory of SIPS in the living human brain. The ensemble tractography (Takemura et al. 2016a) yielded bilateral identification of SIPS in all subjects. Evidence for SIPS was evaluated based on the consistency across datasets, comparison with post-mortem fibre dissection studies (Sachs 1892; Vergani et al. 2014), and the virtual lesion analysis (Pestilli et al. 2014; Leong et al. 2016; Gomez et al. 2015; Takemura et al. 2016b). We also explored the functional relevance of SIPS by performing fMRI experiments on the same subjects and examining the spatial proximity between the SIPS endpoints and functionally defined cortical regions previously associated with optic-flow processing (Cardin and Smith, 2010, 2011; Greenlee et al. 2016). Results showed that the dorso-lateral SIPS endpoints are near VIP, PcM and p2v, whereas the ventro-medial SIPS endpoints are near PIC+; placing SIPS in a good position to channel sensory signals between the distant cortical areas underlying visuovestibular integration necessary for optic-flow processing and perception of self-motion. Finally, we also demonstrate evidence of SIPS in additional 90 subjects from publically available Human Connectome Project datasets.

For the first time in the living human brain, we describe human SPIS in a manner consistent with the previous postmortem dissection studies. Our findings confirm that SIPS is a short-range tract connecting the superior and inferior parts of the parietal cortex, wrapping around the intraparietal sulcus. The findings also highlight that in vivo identification and characterisation of SIPS open new avenues to studying this tract in relation to cortical functions, their development, and diseases that affect them.

To facilitate future research, we make the code to identify human SIPS publicly available in Github repository [https://github.com/htakemur/SIPS] and also as part of AFQ toolbox [Yeatman et al. 2012b; https://github.com/ yeatmanlab/AFQ].

\section{Materials and methods}

We analysed dMRI data of 100 human subjects, from three independent datasets. One set of dMRI data was acquired at Kokoro Research Center, Kyoto University (KU dataset), 
along with fMRI measurements to identify cortical regions activated by optic-flow stimulation. The other dMRI datasets were obtained from the Human Connectome Project (HCP) by WU-Minn Consortium (WU-Minn HCP; Van Essen et al. 2013) and MGH-USC Consortium (MGHUSC HCP; Fan et al. 2016).

\section{Subjects: KU dataset}

Six healthy volunteers (three males and three females; of the ages between 22 and 47 years; subjects S1-S6) participated in the study. All six subjects underwent both dMRI and fMRI experiments. All had normal or correctedto-normal vision. All individual subjects gave written informed consent to take part in this study, which was conducted in accordance with the ethical standards stated in the Declaration of Helsinki and approved by the local ethics and safety committees at Kyoto University.

\section{Data acquisition and preprocessing: $\mathrm{KU}$ dataset}

All MR images were obtained with a 3-T Siemens Magnetom Verio scanner (Siemens, Erlangen, Germany), using a Siemens 32-channel head coil, at Kokoro Research Center, Kyoto University.

\section{Diffusion-weighted MRI data}

Two repeated acquisitions of MR images were conducted for each subject, using a diffusion-weighted single-shot spin echo, echo-planar sequence [60 axial slices, 2-mm isotropic voxels, time of repetition (TR) $8300 \mathrm{~ms}$, time echo (TE) $94 \mathrm{~ms}$, field of view (FoV) $200 \times 200 \mathrm{~mm}^{2}$ ]. The dMRI data were sampled in 64 directions with a $b$ value of $1000 \mathrm{~s} / \mathrm{mm}^{2}$. Two non-diffusion-weighted images $(b=0)$ were obtained.

Diffusion data were preprocessed using mrDiffusion, implemented in Matlab as part of the mrVista software distribution (https://github.com/vistalab/vistasoft). MR images in each scan were motion-corrected to the $b=0$ image acquired in the same scan, using a rigid-body alignment algorithm implemented in SPM (Friston and Ashburner 2004). Eddy current correction and head-motion correction were applied in the process of 14-parameter constrained nonlinear coregistration, based on the expected patterns of eddy current distortion given the phase-encoding directions of the acquired data (Rohde et al. 2004). The gradient direction in each diffusion-weighted volume was corrected using the rotation parameters from the motion correction procedure. Subsequently, fibre orientation in each voxel was estimated using constrained spherical deconvolution (CSD; Tournier et al. 2007; $L_{\max }=8$ ) implemented in MRtrix (Tournier et al. 2012). CSD allows for tractography based on a model that is capable of accounting for crossing fibres. Because the tract connecting the superior and inferior parts of the parietal cortex likely intersects with the neighbouring major fasciculi such as the arcuate fasciculus, CSD was employed to fully reconstruct the tract.

\section{T1-weighted image data}

For each subject, a high-resolution T1-weighted 3D anatomical image was acquired with a magnetisation-prepared rapid-acquisition gradient echo (MP-RAGE; 208 axial slices, 1-mm isotropic voxels, TR $2250 \mathrm{~ms}$, TE $3.51 \mathrm{~ms}$, FoV $256 \times 256 \mathrm{~mm}^{2}$, flip angle $9^{\circ}$, bandwidth $230 \mathrm{~Hz} /$ pixel), and the border between the grey matter and white matter was defined. Initial segmentation was performed using an automated procedure in Freesurfer (Fischl 2012), which was then refined manually (Yushkevich et al. 2006).

\section{Functional data and localisation of optic-flow selective} sensory regions

To determine the spatial relations between the white matter tract and the optic-flow selective sensory areas (Cardin and Smith 2011; Frank et al. 2014), four regions of interest (ROIs) were identified with data acquired in separate fMRI localiser scans, using the procedure described in Uesaki and Ashida (2015). The stimulus was a random dot kinematogram consisting of 200 moving light dots of $10 \times 10$ pixels (subtending approximately $0.4^{\circ} \times 0.4^{\circ}$ visual angle) on a dark background. The dots initially appeared at random locations and formed a circular patch of $30^{\circ}$ diameter. Motion directions of the dots were arranged so that the dots (a) cycled through a spiral space with time-varying trajectories away from and towards the centre of the patch for the optic-flow stimulus, or (b) moved in random directions for the random-motion stimulus. The coherent optic-flow and random-motion stimuli were presented in 16-s blocks. Subjects maintained central fixation throughout the experiment. No attentional task was undertaken.

Functional data were acquired with a gradient echo, echo-planar sequence (39 contiguous axial slices, 3-mm isotropic voxels, TR $2000 \mathrm{~ms}$, TE $25 \mathrm{~ms}$, FoV $192 \times 192 \mathrm{~mm}^{2}$, flip angle $80^{\circ}$, bandwidth $2368 \mathrm{~Hz} /$ pixel), using a Siemens 32-channel posterior-head array coil; which gave an improved signal-to-noise ratio in the occipital cortex at the expense of the anterior part of the brain. Each subject underwent two fMRI scans. Functional data were then preprocessed and analysed with BrainVoyager QX (version 2.6, Brain Innovation, Maastricht, The Netherlands). Analysis was conducted by fitting a general linear model (GLM). Each of the 16-s stimulus 
blocks was convolved with a canonical haemodynamic response function, and entered into a multiple-regression analysis to generate parameter estimates for each regressor at each voxel. Blood-oxygen-level-dependent responses to the coherent optic-flow and random-motion stimuli were contrasted, which allowed for isolation of cortical regions that are significantly more sensitive to coherent optic flow at $p$ value (uncorrected) of less than 0.005 . The analysis was performed on the $3 \mathrm{D}$ anatomical volumes for each subject. The ROIs were defined as all contiguous voxels that were significantly more active with coherent opticflow stimulation than with random-motion stimulation (Uesaki and Ashida 2015); in the ventral intra-parietal cortex (VIP), the precuneus motion area (PcM), the junction of the intra-parietal sulcus $(\mathrm{p} 2 \mathrm{v})$, and the posterior region of the insular cortex $(\mathrm{PIC}+)$. Stimulus design and analysis methods are described in more detail elsewhere (Uesaki and Ashida 2015).

\section{Data acquisition and preprocessing: Human Connectome Project datasets}

Diffusion-weighted MRI data obtained from 61 subjects in WU-Minn HCP dataset (S7-S67: Van Essen et al. 2013) and 33 subjects in MGH-USC HCP dataset (S68-S100: Fan et al. 2016) were also analysed in this study. These data were acquired with multiple $b$ values (1000, 2000 and $3000 \mathrm{~s} / \mathrm{mm}^{2}$ for WU-Minn HCP dataset; 1000, 3000, 5000 and $10,000 \mathrm{~s} / \mathrm{mm}^{2}$ for MGH-USC HCP dataset), high spatial $\left(1.25 \times 1.25 \times 1.25 \mathrm{~mm}^{3}\right.$ for WU-Minn HCP dataset; $1.5 \times 1.5 \times 1.5 \mathrm{~mm}^{3}$ for MGH-USC HCP dataset) and angular resolution (90 directions at each $b$ value for WU-Minn HCP dataset; 64 directions at $b=1000,3000 \mathrm{~s} /$ $\mathrm{mm}^{2}$ and 128 directions at $b=5000,10,000 \mathrm{~s} / \mathrm{mm}^{2}$ for MGH-USC HCP dataset). We note that the selected HCP data were acquired with greater spatial and angular resolution, and higher $b$ values compared with KU dataset. All HCP data were preprocessed by WU-Minn HCP and MGH-USC HCP Consortiums using methods that are described in Sotiropoulos et al. (2013) and Fan et al. (2014), respectively.

\section{Data analysis}

\section{Coregistration of functional and diffusion MR images to T1-weighted images}

T1-weighted 3D anatomical images were aligned to the anterior commissure-posterior commissure (AC-PC) plane. To do this, the locations of AC and PC were manually defined in the T1-weighted images. These landmarks were then used to apply rigid-body transformation to align the anatomical images to the $\mathrm{AC}-\mathrm{PC}$ plane.
Preprocessed fMRI and dMRI data were coregistered to the T1-weighted images in the AC-PC space, using a twostage coarse-to-fine approach. This process enabled a direct spatial comparison between the tract endpoints and the ROIs within the same coordinates in each subject, in the later analyses.

\section{Tractography}

We used two complementary approaches to perform tractography. One is the ensemble tractography (Takemura et al. 2016a) based on the linear fascicle evaluation (LiFE; Pestilli et al. 2014; http://francopestilli.github.io/life/), which generates streamlines with various tractography parameters and removes those that do not contribute to predicting the diffusion signals. The advantage of this approach is that spurious streamlines that do not explain diffusion signals are not included in the resulting model. It also allows for evaluation of statistical significance of the estimated tract based on cross-validation (virtual lesion analysis; Pestilli et al. 2014; Takemura et al. 2016b; Leong et al. 2016). However, this approach requires a large amount of computational resources to optimise large-scale linear matrices composed of diffusion signals in every voxel acquired with multiple angular directions, as well as a mass of candidate streamlines (Takemura et al. 2016a). This makes it less practical to apply this framework to analysing data from large samples, until the ongoing work to reduce the necessary computational load of LiFE is completed (Caiafa and Pestilli 2017). Another limitation is that the current implementation of LiFE only accepts single-shell dMRI data (Pestilli et al. 2014).

The alternative approach is to exclude the ensemble tractography and LiFE from the analysis pipeline. At a cost of opting out statistical evaluation using the identical methods, we analysed the data of a large sample (i.e. 100 subjects) to assess the generality of our findings. Additionally, this alternative approach has a distinct advantage over the ensemble tractography and LiFE analyses, which is that it allows for tracking algorithms based on multi-shell dMRI data (Jeurissen et al. 2014). These two approaches can be complementary in validating the findings; one is designed to evaluate the statistical evidence on smaller samples, whereas the other simpler pipeline is well suited for assessing the generality across a larger number of subjects.

Ensemble tractography For the six subjects of KU dataset (S1-S6) and four subjects from WU-Minn HCP dataset (S7-S10), we used the ensemble tractography (Takemura et al. 2016a) to estimate the white matter tract based on dMRI data from each subject. Measurements from the $2000 \mathrm{~s} / \mathrm{mm}^{2}$ shell were extracted from the original WUMinn HCP dataset and used for analyses. 
Candidate streamlines were generated with MRtrix (Tournier et al. 2012), using CSD-based probabilistic tractography (step size $0.2 \mathrm{~mm}$; maximum length $200 \mathrm{~mm}$; minimum length $10 \mathrm{~mm}$; fibre orientation distribution (FOD) amplitude stopping criterion 0.1). We used the entire grey matter-white matter interface region as a seed (Smith et al. 2012a), and the seed voxels were randomly selected from the mask for generating candidate streamlines. Tracking was terminated when a streamline reached outside the white matter mask. We used four different angular threshold settings (angular threshold $5.7^{\circ}, 11.5^{\circ}$, $23.1^{\circ}, 47.2^{\circ}$ ). Two million candidate streamlines were generated for each curvature parameter setting. We then selected those located within the white matter posterior to the most anterior optic-flow selective area (the cingulate sulcus visual area; CSv; Cardin and Smith 2010; Smith et al. 2012b, 2017) in each hemisphere, which were used in all subsequent analyses.

To obtain an optimised connectome model comprising streamlines generated with different curvature thresholds, we used the preselection method of the ensemble tractography (Takemura et al. 2016a). First, we separately optimised each connectome generated with a single curvature parameter using LiFE. We then selected 150,000 streamlines that contributed meaningfully to predicting the diffusion signals from each single-parameter connectome, and combined them to create a new candidate connectome (the ensemble tractography connectome; ETC; 600,000 candidate streamlines) in each hemisphere. Finally, LiFE was applied again to optimise the ETC. As a result, the optimised ETC included 103,664 streamlines on average per hemisphere. The optimised ETC has been shown to perform better than conventional connectome models, in terms of model accuracy and anatomical representation (Takemura et al. 2016a; https://github.com/brain-life/ensemble_ tractography). Optimised ETCs were used for identification and statistical evaluation of SIPS.

Tractography analysis of a large sample For dMRI data of the other 90 subjects (56 subjects from WU-Minn HCP and 34 subjects from MGH-USC HCP datasets), we performed probabilistic tractography based on multi-tissue CSD implemented in MRtrix (Jeurissen et al. 2014; step size $0.625 \mathrm{~mm}$ for WU-Minn $\mathrm{HCP}, 0.75 \mathrm{~mm}$ for MGHUSC HCP; angular threshold $45^{\circ}$; minimum length $2.5 \mathrm{~mm}$ for WU-Minn HCP, $3 \mathrm{~mm}$ for MGH-USC HCP; maximum length $250 \mathrm{~mm}$; FOD amplitude stopping criterion 0.1). Measurements of all $b$ values are used for estimating multitissue CSD. We used the entire grey matter-white matter interface region as a seed (Smith et al. 2012a), and the seed voxels were randomly selected from the mask for generating candidate streamlines. Tracking was terminated when a streamline reached the white matter mask. As a result, two million streamlines were generated. LiFE was not applied in this analysis.

\section{Tract segmentation}

We used the cortical ROIs defined by Freesurfer segmentation (Desikan-Killiany atlas; Desikan et al. 2006) to identify the white matter tract connecting the superior and inferior parts of the parietal cortex in the optimised connectome. For the main analysis, two cortical ROIs, one in the superior parietal cortex and the other in the inferior parietal cortex were created. The ROI in the superior part of the parietal cortex was defined as the combination of two Freesurfer ROIs: "precuneus" and "superior_parietal". The combination of those two ROIs covered the positions of the functionally identified areas located in the superior parietal cortex (VIP, PcM, p2v). The Freesurfer ROI-labelled "supramarginal gyrus" was used as the ROI in the inferior parietal cortex, which covered the general region including PIC + .

The tract between the superior and inferior parts of the parietal cortex was identified as a group of streamlines having one of the endpoints near the superior parietal ROI and the other near the inferior parietal ROI (within $3 \mathrm{~mm}$ from each grey matter ROI; i.e. within two voxels from each grey matter ROI in KU, MGH-USC HCP datasets and three voxels in WU-Minn HCP dataset). Only streamlines terminating near the grey matter ROIs and not those passing through the grey matter ROIs were included, since tractography was anatomically constrained by the grey matter-white matter interface region used as a seed in generating candidate streamlines and did not allow for streamlines passing through the grey matter-white matter boundary to be generated (Smith et al. 2012a). The segmented tract was then refined by removing outlier streamlines. Those were streamlines that met the following criteria: (1) streamlines longer than the mean streamline length of the tract by $\geq 3 \mathrm{SD}$; (2) streamlines shorter than $15 \mathrm{~mm}$; (3) streamlines of which positions are $\geq 3$ SD away from the mean position of the tract (Yeatman et al. 2012b).

The MATLAB code used to identify SIPS in this study is available in Github repository [https://github.com/htake mur/SIPS] and also as part of the AFQ toolbox [Yeatman et al. 2012b; https://github.com/yeatmanlab/AFQ].

\section{Estimating cortical endpoints of the tract}

Streamlines terminate at the boundary between the white matter and grey matter. To estimate the SIPS endpoints in the cortical surface representation, the coordinates of the SIPS endpoints were collected, and the distance between those coordinates and the grey matter voxels was calculated. For each grey matter voxel, the number of the SIPS 
endpoints falling within a threshold distance $(3 \mathrm{~mm})$ was counted. The normalised endpoint counts are plotted on the inflated cortical surface in Fig. 1b. The same method was used in Takemura et al. (2016b).

\section{Virtual lesion analysis}

We conducted the virtual lesion analysis (Pestilli et al. 2014; Leong et al. 2016; Takemura et al. 2016b) on KU dataset to evaluate the statistical evidence supporting the existence of the white matter tract connecting the superior and inferior parts of the parietal cortex. For this analysis, we divided the dMRI data (KU dataset) into two sessions; one was used for performing tractography, and the other was used for computing cross-validated model prediction accuracy.

Two connectome models were used in the analysis; the optimised connectome and a lesioned connectome with the streamlines of interest (i.e. the streamlines that belong to the tract connecting the superior and inferior parts of the parietal cortex) removed. We computed prediction accuracy (root-mean-squared error; RMSE) of those models in predicting the diffusion signals. The set of dMRI data from the second session was used as the measured diffusion signals for cross-validation. Model accuracy is described as a ratio of RMSE $\left(R_{\text {rmse }}\right)$, and it represents prediction accuracy of each model with respect to test-retest reliability (for calculation methods, see Rokem et al. 2015; Takemura et al. 2016a). $R_{\text {rmse }}=1$ indicates that the model accuracy for predicting the diffusion signals in the second dataset equals to test-retest reliability of the diffusion signals in the same voxel.

$R_{\text {rmse }}$ was compared in all voxels touched by the lesioned streamlines (the streamlines that belong to SIPS). The complete set of streamlines that contribute to the prediction of the diffusion measurements in those voxels is called the path neighbourhood of SIPS (Wedeen et al. 2012). This path-neighbourhood includes SIPS itself and all of the other streamlines that pass through the voxels SIPS passes through. We calculated the distribution of $R_{\text {rmse }}$ values in SIPS voxels with the entire path-neighbourhood included and removed SIPS, to figure out the weight of each of the remaining streamlines.

Finally, we compared the two $R_{\text {rmse }}$ distributions using the strength of evidence, $S$, which is the difference in the mean $R_{\text {rmse }}$ divided by the joint standard deviation (for technical detail; see Pestilli et al. 2014).

\section{Spatial proximity between SIPS endpoints and functionally defined ROIs}

To characterise the spatial proximity between the opticflow selective ROIs and SIPS, we measured the proportion of grey matter voxels in each ROI (VIP, PcM, p2v and $\mathrm{PIC}+$ ) located adjacent to the SIPS endpoints. We computed the three-dimensional distance between the endpoints of each SIPS streamline and each grey matter voxel included in the ROIs. We then calculated the proportion of voxels in each ROI located within a specific distance (thresholded at 3 or $4.5 \mathrm{~mm}$ in volumetric space) from any SIPS endpoints. This procedure is based on that reported in Takemura et al. (2016b).

We note that there are limitations to this analysis, due to the general difficulty in associating streamline endpoints and the grey matter surface (Reveley et al. 2015), particularly for dMRI data with a standard resolution such as those used in this study (i.e. KU dataset). The aim of this analysis was to examine the general spatial proximity between the tract endpoints and functionally defined ROIs, rather than to determine the projection pattern of the tract on the cortical surface.

\section{Probabilistic atlas of SIPS}

We created the probabilistic atlas of SIPS based on our 100 -subject dataset following the method proposed in previous works (Bürgel et al. 2006; Catani and Thiebaut de Schotten 2012). The $b=0$ images of each subject were normalised to the Montreal Neurological Institute 152 (MNI152) space using the MNI152 EPI template to obtain the affine transformation matrix. We then generated a binary visitation map of SIPS in each subject by assigning a value of 1 or 0 to each voxel depending on whether the voxel is intersected by any SIPS streamlines (Catani et al. 2007; Thiebaut de Schotten et al. 2008). This binary visitation map was normalised to MNI152 space according to the transformation matrix derived from the normalisation of the $b=0$ images. Finally, we computed the percentage overlap by summing the normalised visitation maps of all subjects at each point in MNI152 space (Bürgel et al. 2006; Catani and Thiebaut de Schotten 2012). Supplementary Figures 2-5 show the visualisation of the percentage overlap with the overlap threshold at greater than $25 \%$ of the sample.

\section{Results}

The primary aim of this study was to identify the white matter tract connecting the superior and inferior parts of the parietal cortex. By performing tractography on the dMRI data, we successfully identified SIPS in all subjects. We compared the tractography estimates with SIPS reported in the post-mortem fibre dissection studies (Sachs 1892; Vergani et al. 2014), and evaluated the statistical evidence for the estimates (Pestilli et al. 2014) as well as consistency 
Fig. 1 Human stratum

proprium of interparietal sulcus (SIPS) estimated by

tractography performed on dMRI data (KU dataset).

a Coronal view of SIPS (red) in six subjects (S1-S6; KU dataset), identified by tractography (see "Materials and methods"). Scale bar (white) in the S1 panel indicates $10 \mathrm{~mm}$. The background anatomical (T1-weighted) slice is located immediately anterior to the position of SIPS. $L S$ lateral sulcus. b SIPS endpoints overlaid on the cortical surface (S1, left hemisphere). The spatial distance between SIPS endpoints and grey matter voxels was calculated to plot the number of SIPS streamlines having endpoints close to grey matter voxels (see "Materials and methods"). $C S$ central sulcus, PoCS postcentral sulcus, IPS intraparietal sulcus, STS superior temporal sulcus, CingS cingulate sulcus

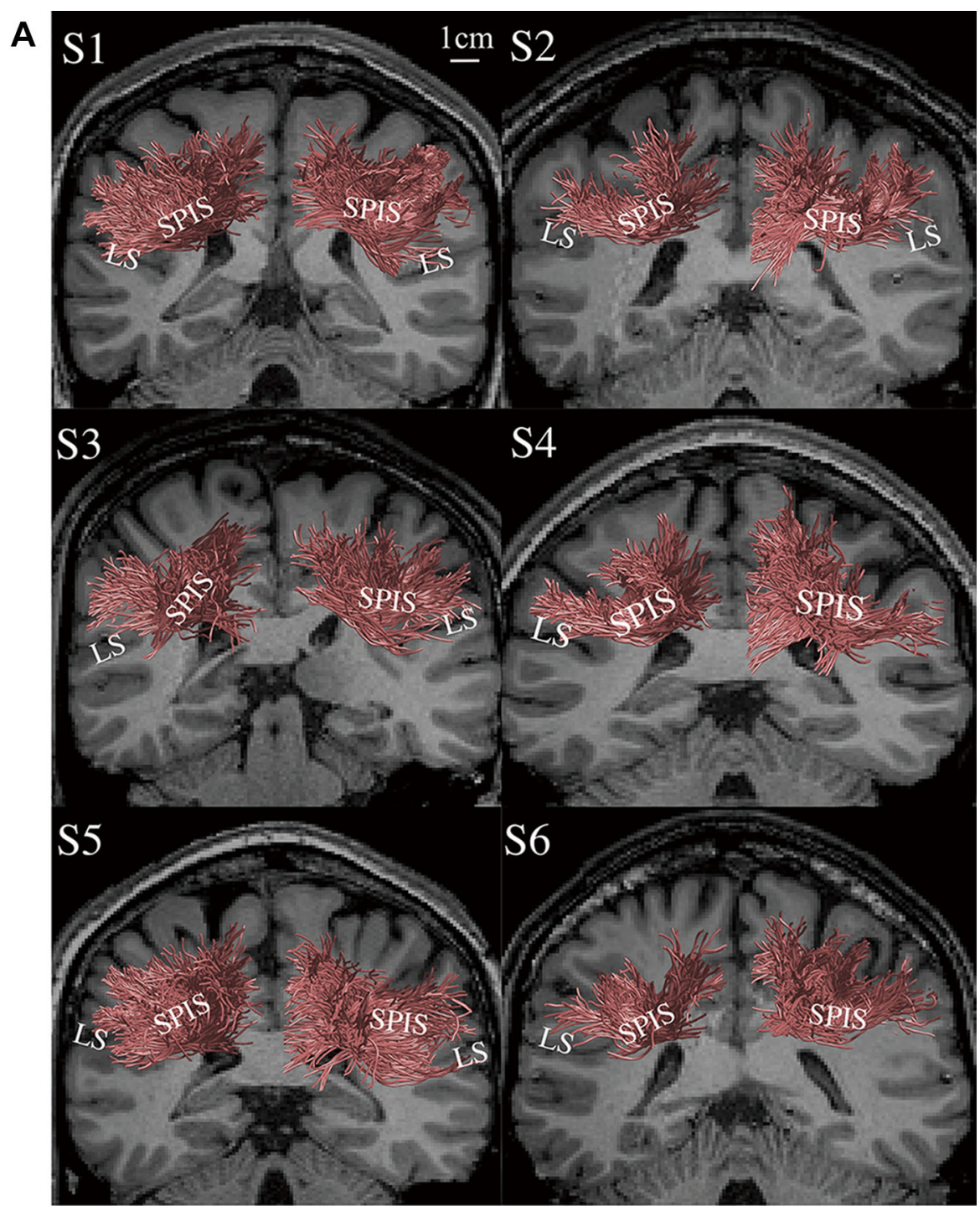

B

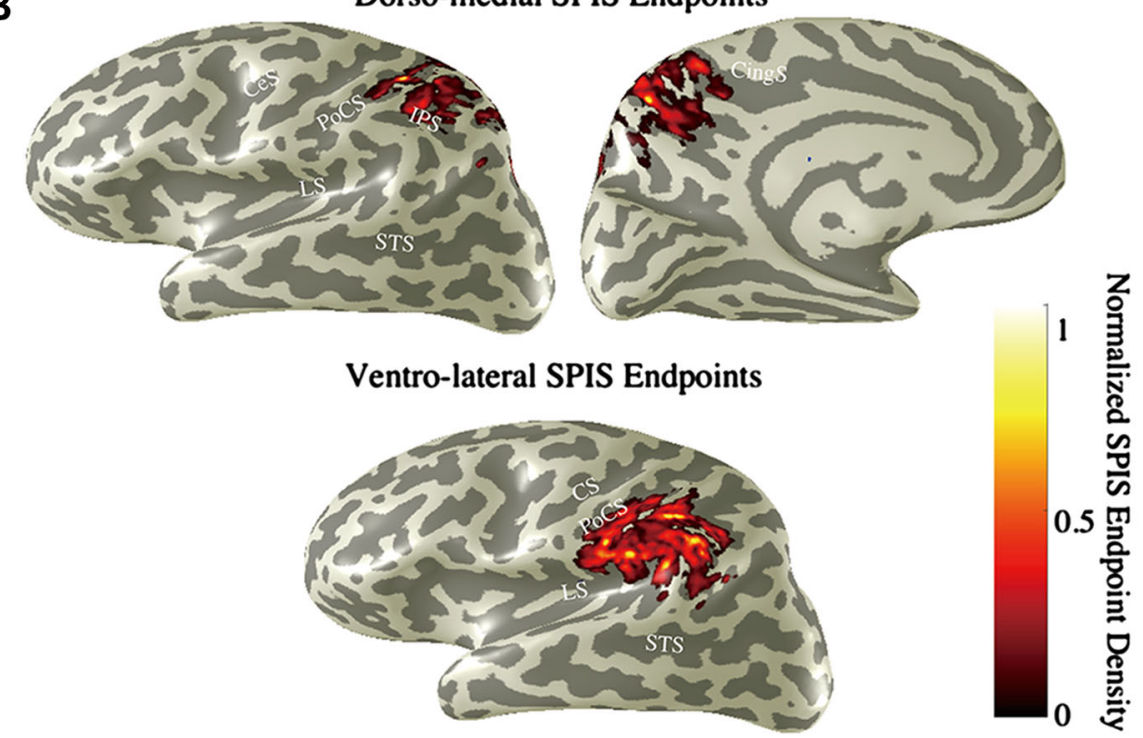


across datasets. The tract was identified consistently across subjects and across datasets, and seems to correspond with the tract referred to as SIPS in previous fibre dissection studies (Sachs 1892; Vergani et al. 2014). Furthermore, we assessed the spatial relations between SIPS and the opticflow selective sensory areas localised using fMRI.

\section{Anatomy of human SIPS}

\section{Tract trajectory and length}

We analysed dMRI data of the six subjects from KU dataset using the ensemble tractography (Takemura et al. 2016a; see "Materials and methods"), whereby streamlines were generated using multiple parameter settings, and then optimised using LiFE (Pestilli et al. 2014; see "Materials and methods"). We identified a white matter tract having one set of endpoints near the superior parietal cortex and another near the inferior parietal cortex, based on the grey matter ROIs defined by Freesurfer (Desikan et al. 2006; Fischl 2012; see "Materials and methods").

Figure 1a shows the group of streamlines that comprises the tract we identified in each hemisphere in six subjects from KU dataset, superimposed on a coronal slice of the T1-weighted anatomical image. The estimated tract, SIPS, primarily connects the superior and inferior parts of the parietal cortex and wraps around the intraparietal sulcus (IPS). In all subjects, SIPS in the two hemispheres are symmetrically oriented. The mean length of the estimated SIPS streamlines was $4.69 \mathrm{~cm}$ (SD $0.59, N=12$ hemispheres). Figure $1 b$ shows the estimated SIPS endpoints on the cortical surface representation in one representative hemisphere (S1, left hemisphere). Most of the dorso-medial SIPS endpoints are not only in the parietal areas superior to the IPS but also in the medial areas such as the precuneus. The ventro-lateral endpoints of SIPS are in the supramarginal gyrus, parietal operculum and the posterior end of the lateral sulcus.

Additionally, we analysed the publicly available HCP data (WU-Minn HCP dataset; Van Essen et al. 2013) to assess the consistency of SIPS results across datasets. Figure 2a demonstrates SIPS in four subjects from WUMinn HCP dataset, identified using the identical methods to those used for $\mathrm{KU}$ dataset. The estimated position and trajectory of SIPS are consistent with those in KU dataset (Fig. 1a).

Figure $2 b$ shows a principal diffusion direction (PDD) map illustrating the position of SIPS in one representative WU-Minn HCP subject (S7). PDD is often used to identify the major white matter tracts, and it allows for tract identification independent of the selection of tractography methods (Pajevic and Pierpaoli 1999; Wakana et al. 2004; Yeatman et al. 2013; Takemura et al. 2016b). This PDD map based on WU-Minn HCP dataset clearly shows the existence of a tract travelling between the medial side of superior parietal cortex, and lateral inferior regions around parietal operculum and posterior part of lateral sulcus.

To assess the generality of our findings, we analysed dMRI data of 90 subjects from WU-Minn HCP and MGHUSC HCP datasets using a simpler analysis pipeline (see "Materials and methods"). Supplementary Figure 1 demonstrates that SIPS was also consistently observed in those subjects. We also describe the population average of the position of SIPS in MNI152 space in Supplementary Figures 2-5.

The results of tractography, which is consistent across a large number of subjects and three independent datasets, as well as voxelwise evidence of SIPS that is not based on tractography, further corroborate the evidence for SIPS. Furthermore, SIPS described here is consistent with a short parietal association bundle reported in a series of white matter atlas works (Oishi et al. 2008, 2011; Zhang et al. 2010; Guevara et al. 2017). However, the atlas does not provide additional information regarding the provenance of SIPS, making our study the first to report the detailed anatomical characteristics of this tract using in vivo dMRI methods and to compare SIPS identified based on in vivo dMRI data to post-mortem findings, as described below.

\section{Comparison with fibre dissection studies}

We compared the anatomical position and shape of SIPS identified from dMRI data with post-mortem fibre dissection studies. SIPS has been documented in two previous post-mortem fibre dissection studies; in the classical work by a German neurologist Heinrich Sachs (1892) and more recently by Vergani et al. (2014). Sachs referred to this tract as stratum proprium fissurae interparietalis in his report, which was later rephrased by Vergani et al. (2014) as stratum proprium of interparietal sulcus (SIPS). We adopt this term, SIPS, to refer to the tract estimated in the present study.

Figure 3 compares the position of SIPS in Sachs's study (1892; Fig. 3a, b) and in Vergani's study (2014; Fig. 3c), with the tract we identified using tractography (Fig. 3d). SIPS identified in this study is consistent with SIPS reported in the fibre dissection studies in several aspects. In terms of its spatial relations with cortical landmarks, SIPS wraps around the intraparietal sulcus, and connects the parietal cortex and the dorsal bank of the lateral sulcus. The position of SIPS on the coronal slice is also consistent. The coronal slice of the anatomical image onto which SIPS estimated by tractography is superimposed in Fig. 3d was chosen carefully so that it corresponded with the slices used in the fibre dissection studies as closely as possible. Whilst it is not possible to perfectly match the position of 
Fig. 2 SIPS identified in HCP dataset. a Coronal view of SIPS (red) in four subjects (S7-S10) from HCP dataset, identified by tractography (see "Materials and methods"). The conventions are identical to those in Fig. 1a. b Position of SIPS highlighted in PDD map (S7, two representative coronal slices; the position of each slice is shown in ACPC coordinate). The colour scheme depicts the PDD in each voxel (blue superior-inferior; green anterior-posterior; red leftright). White matter portion connecting the dorso-medial and ventro-lateral regions wrapping around the intraparietal sulcus (IPS) that is predominantly blue/purple clearly illustrates the trajectory of SIPS. Yellow-dotted line highlights the position of SIPS
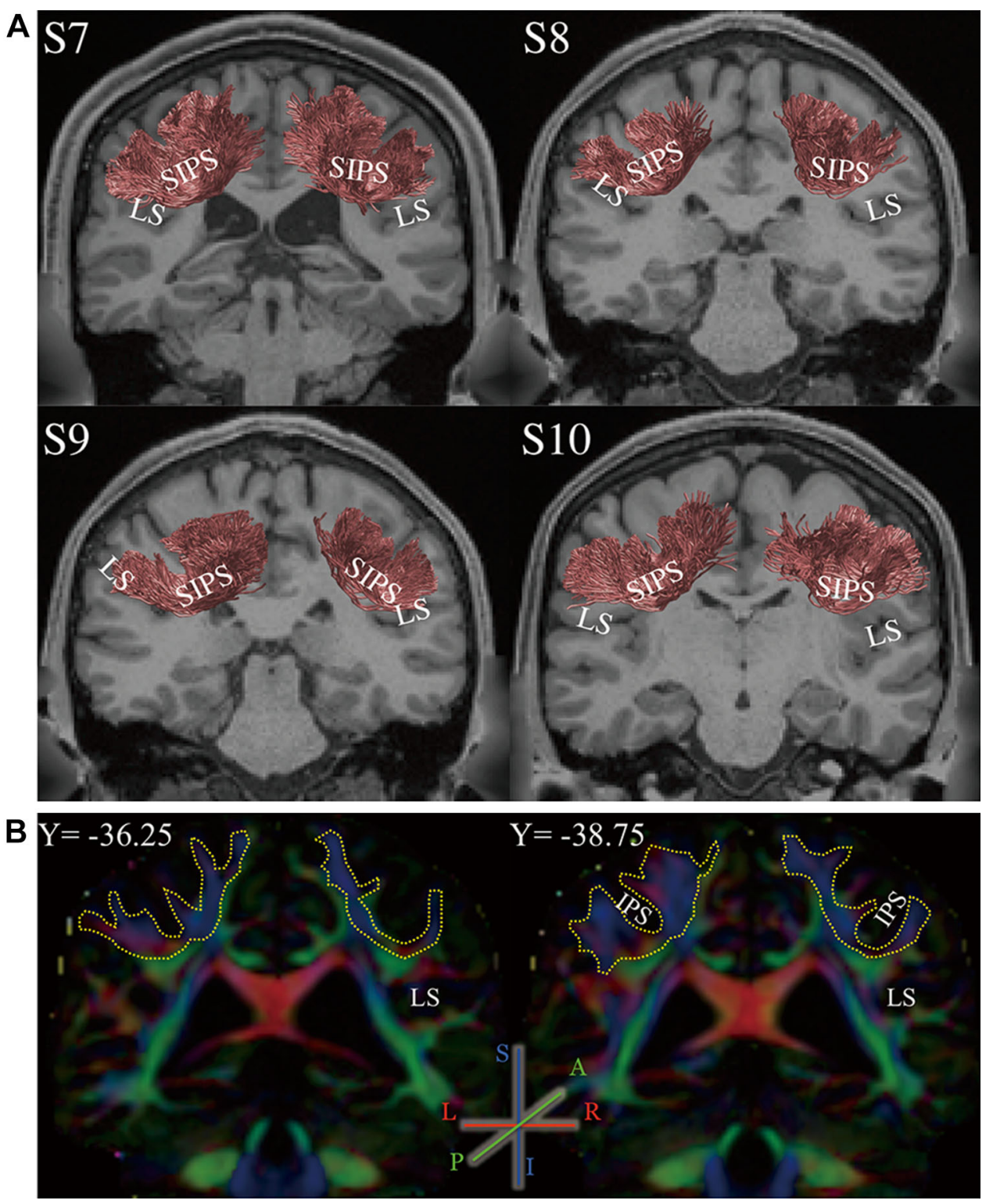

the slice between our MRI data and fibre dissection studies, it is qualitatively consistent across the four presentations in Fig. 3, in terms of the positions of the sulci (i.e. the lateral sulcus and the intraparietal sulcus) and the lateral ventricle. Although SIPS in the three studies cannot be compared quantitatively due to the difference in the methodology used, Fig. 3 highlights that the position and trajectory of SIPS identified with our dMRI data agree with those of SIPS reported in the fibre dissection studies.

\section{Position of SIPS with respect to major white matter tracts}

Figure 4 shows SIPS overlaid on a sagittal plane of the T1weighted image, along with other major white matter tracts reported in previous studies; the arcuate fasciculus (AF;
Catani et al. 2002; Wakana et al. 2004), posterior arcuate (pArc; Catani et al. 2005; Weiner et al. 2016) and vertical occipital fasciculus (Yeatman et al. 2013, 2014b; Duan et al. 2015; Takemura et al. 2016b) in one representative subject (S1).

SIPS is located adjacent to AF, and in fact, SIPS intersects with the dorsal surface of AF. This crossing may be one of the reasons that this tract has been relatively neglected in the literature, as resolving crossing fibres is one of the critical limitations of the diffusion tensor-based approach (Frank 2001; Tournier et al. 2012). Interestingly, the intersection between SIPS and AF may explain the pattern of previous dMRI results along AF. Yeatman et al. (2011) investigated the fractional anisotropy (FA; Basser and Pierpaoli 1996) along AF, and found that there is a 

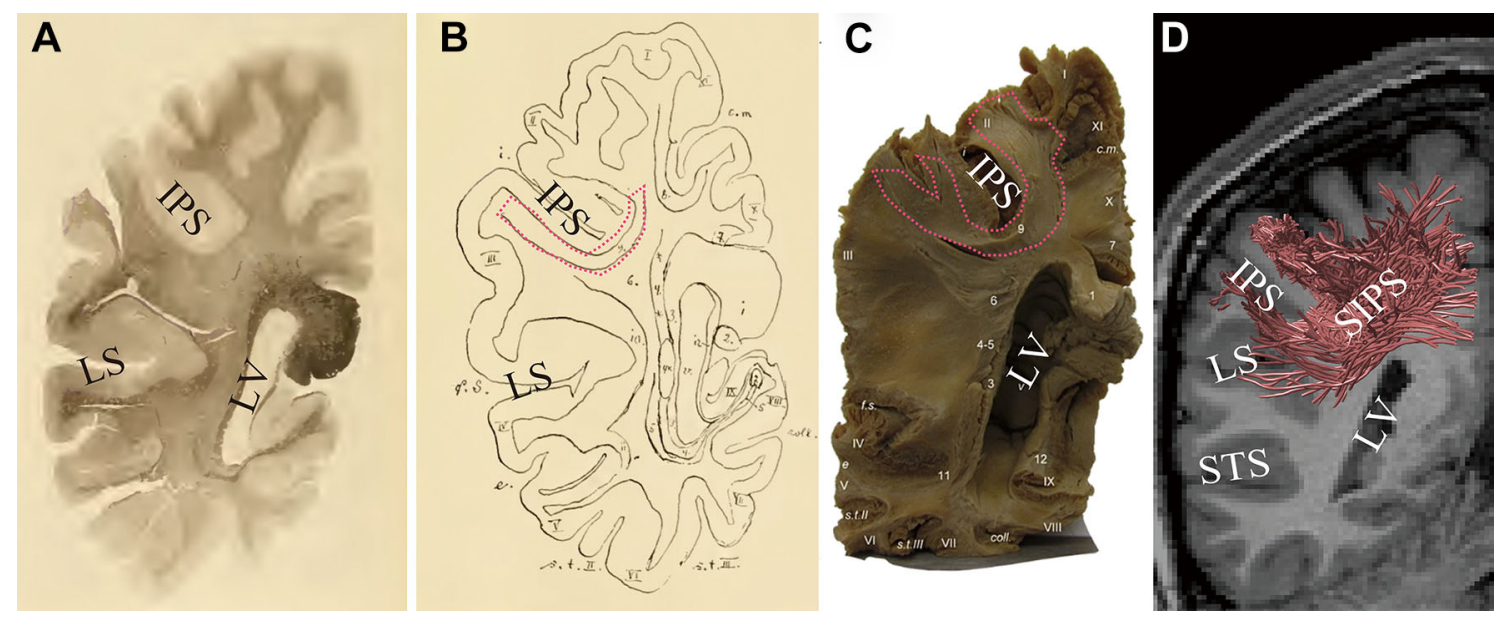

Fig. 3 Tractography results are consistent with classical and modern fibre dissection studies. a White matter tract referred to as SIPS on a coronal histological slice and b SIPS (red outline) in the schematic diagram of the fibres visualised in the photo in a (Sachs 1892). Sachs (1892) noted that this slice is approximately $75 \mathrm{~mm}$ anterior to the occipital pole (Forkel et al. 2015). c SIPS identified in a post-mortem human brain in the modern fibre dissection study (right hemisphere; Vergani et al. 2014). The position of SIPS is highlighted with red outline. Reproduced from Vergani et al. (2014) with permission. d SIPS estimated by tractography (red lines) in one representative hemisphere (S1; right hemisphere). The image has been flipped (for the original image, see Fig. 1a) so that the slice corresponds with the fibre dissection studies. The background coronal slice (ACPC coordinate, $Y=-44$; approximately $65 \mathrm{~mm}$ anterior to the occipital pole) is located immediately anterior to the estimated SIPS. The position and the trajectory of SIPS are qualitatively consistent with those of SIPS reported in the fibre dissection studies $(\mathbf{a}-\mathbf{c}) . L V$ lateral ventricle, STS superior temporal suclus, IPS intraparietal sulcus
Fig. 4 Position of SIPS with respect to other tracts. a Position of SIPS with respect to other tracts in the left hemisphere of one representative subject (S1). The background T1-weighted image is a sagittal slice in the medial portion of the brain. SIPS (red) is located superior to the vertical occipital fasciculus (VOF; yellow) and posterior arcuate (pArc; green). SIPS lies on the superior surface of, and crosses with the arcuate fasciculus (arcuate; light blue). b Position of SIPS with respect to other tracts in the right hemisphere in the same subject (S1)

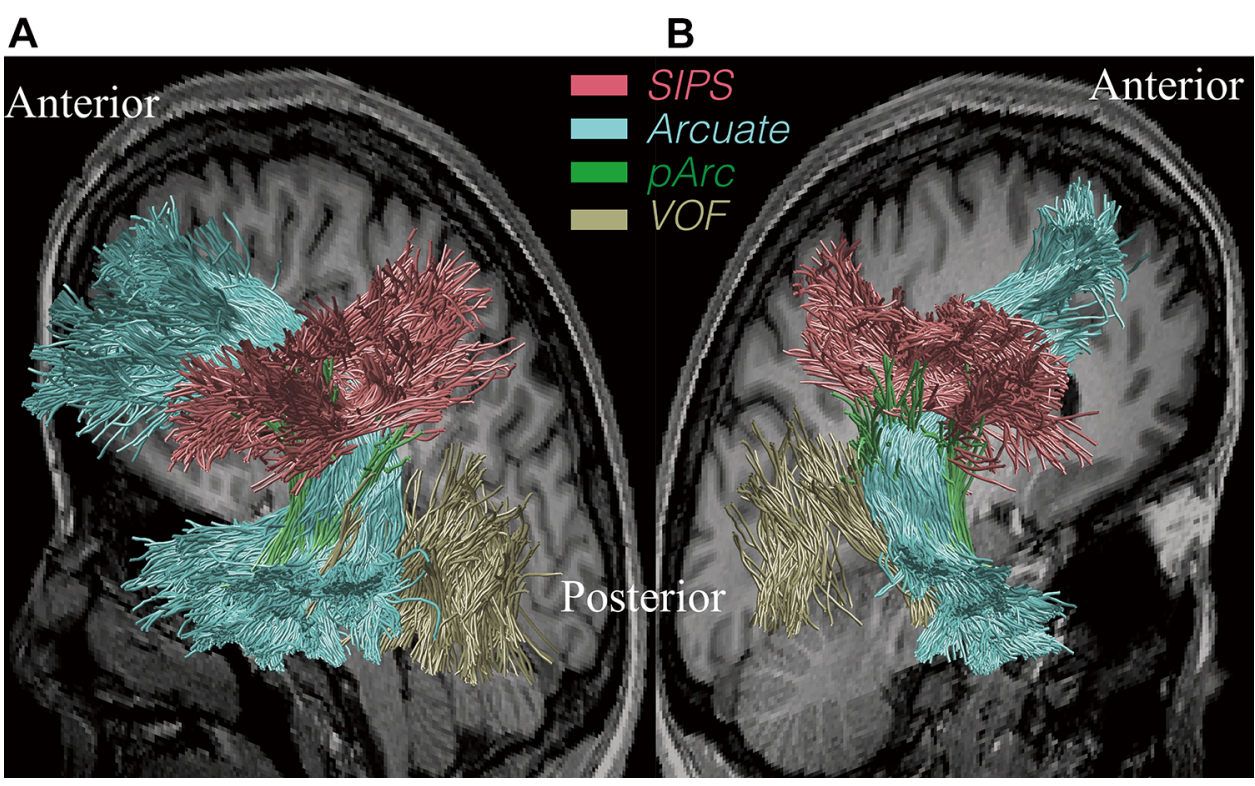

large dip in the FA value along the length of the fasciculus in the vicinity of the temporal cortex. Yeatman et al. (2011) suggest that this dip is not only partially accounted for by the sharp curvature of AF but also by partially voluming with crossing fibres. Since the location of this dip along the trajectory of AF coincides with the position of SIPS, it seems plausible that this is where AF intersects with SIPS.

SIPS is also located near pArc, but the trajectory and endpoints of SIPS are distinct from pArc, which connects the parietal cortex and the anterior inferior temporal cortex.
Although there are a few neighbouring tracts, some of which cross or kiss SIPS, the differences in the trajectory and locations of endpoints between those known tracts and SIPS clarify that SIPS is a distinct tract.

\section{Statistical evidence in support of SIPS}

To evaluate the strength of statistical evidence supporting the existence of SIPS, we used the virtual lesion methods (Honey and Sporns 2008; Pestilli et al. 2014; Leong et al. 
2016; Takemura et al. 2016b). We first computed the crossvalidated prediction accuracy for diffusion signals (ratio of RMSE; $R_{\text {rmse }}$; Rokem et al. 2015; Takemura et al. 2016a) in models with lesioned and unlesioned SIPS. We then compared the distribution of $R_{\text {rmse }}$ of the two models to predict the diffusion signals within SIPS voxels (see "Materials and methods" for details). We quantify the strength of evidence $(S)$ in support of the SIPS by calculating the difference of $R_{\text {rmse }}$ in lesioned and unlesioned models divided by the standard deviation of the $R_{\text {rmse }}$ (Pestilli et al. 2014).

Figure 5a describes the mean and variance of the statistical evidence for SIPS across subjects, yielded by the virtual lesion analysis. The mean strength of statistical evidence for SIPS was $S=76.25$ (SD 10.87) for the left hemisphere, and $S=84.7$ (SD 6.72) for the right hemisphere. Figure $5 \mathrm{~b}$ describes the two-dimensional histogram of $R_{\text {rmse }}$ in the SIPS lesioned and unlesioned models for the left hemisphere in one representative subject (S1). In many voxels, the SIPS-lesioned model showed substantially lower model accuracy (higher $R_{\text {rmse }}$ ) as compared with the unlesioned model, indicating that SIPS is necessary to explain the diffusion signals within those voxels. Thus, in addition to the results of tractography and their consistency with the findings of previous post-mortem studies at visual inspection, there is strong statistical evidence supporting the existence of SIPS.

\section{SIPS and its relations with optic-flow selective cortical areas}

With the subjects in $\mathrm{KU}$ dataset, we further conducted fMRI experiments to localise cortical sensory areas selective for optic-flow stimulation to examine the spatial proximity between the SIPS endpoints and those functionally defined areas.

\section{Functional localisation}

To localise the cortical areas selective for optic-flow stimulation, blood-oxygen-level-dependent (BOLD) responses to the coherent optic-flow stimulus was contrasted against those to the random-motion stimulus. We identified four of the cortical areas known to be selective for optic flow (Fig. 6; VIP, p2v, PcM, PIC+; Cardin and Smith 2011; Uesaki and Ashida 2015). Areas VIP, p2V and $\mathrm{PcM}$ are located in the superior part of the parietal cortex, and PIC + in the posterior end of the lateral sulcus. The locations of those areas in Talairach coordinates were consistent with those of the corresponding areas reported in previous studies (Cardin and Smith 2011; Frank et al. 2014; Uesaki and Ashida 2015). All four areas were successfully identified in nine hemispheres. In the other three hemispheres, either PcM or VIP was not identified.

\section{SIPS endpoints and optic-flow selective cortical areas}

Subsequently, we examined the spatial proximity between the cortical areas selective for optic flow (Fig. 6) and the SIPS endpoints. Although there is a limitation to use tractography for identifying the tract endpoints in the grey matter (Reveley et al. 2015), it is still useful to understand how closely functionally defined ROIs are located to the tract endpoints to infer any potential implication of the tract in information transmission during optic-flow processing. We analysed the general spatial proximity between

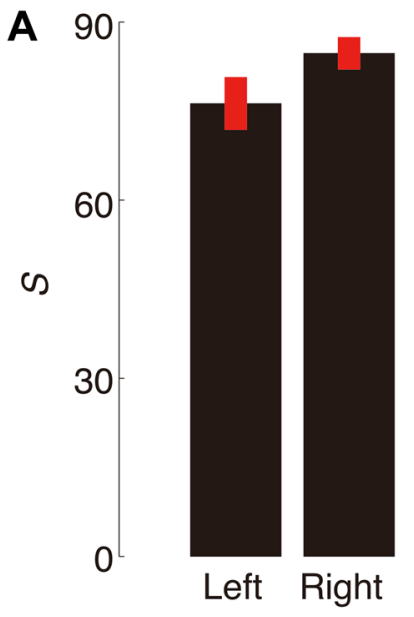

Fig. 5 Statistical evidence in support of SIPS. a Mean $S$ in support of left and right SIPS across subjects. Error bars depict \pm 1 SEM across subjects. b Two-dimensional histogram comparing the model accuracy $\left(R_{\text {rmse }}\right)$ between the lesioned and unlesioned models (horizontal

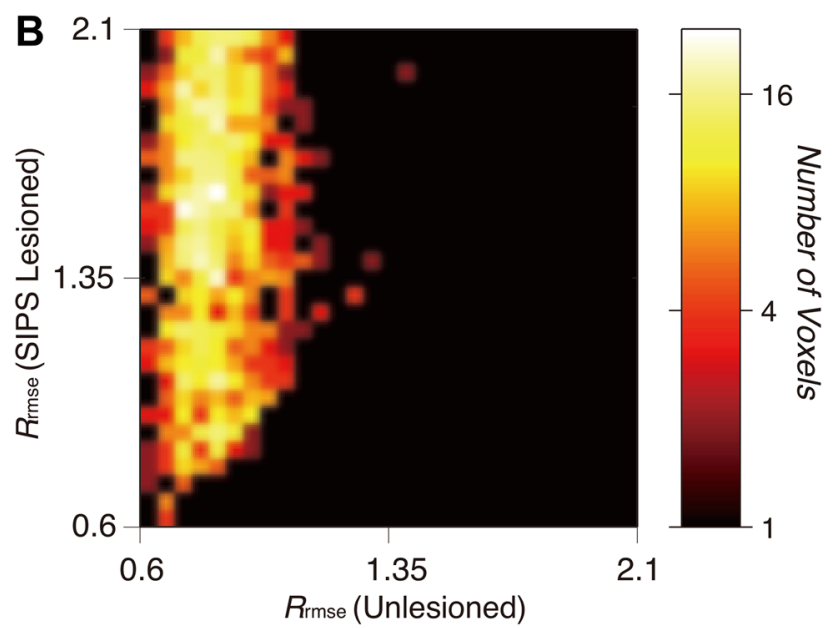

axis unlesioned model; vertical axis lesioned model) for SIPS in the left hemisphere in one representative subject (S1). Prediction accuracy is substantially lower with the lesioned model. Colour bar (right panel) indicates the number of voxels 


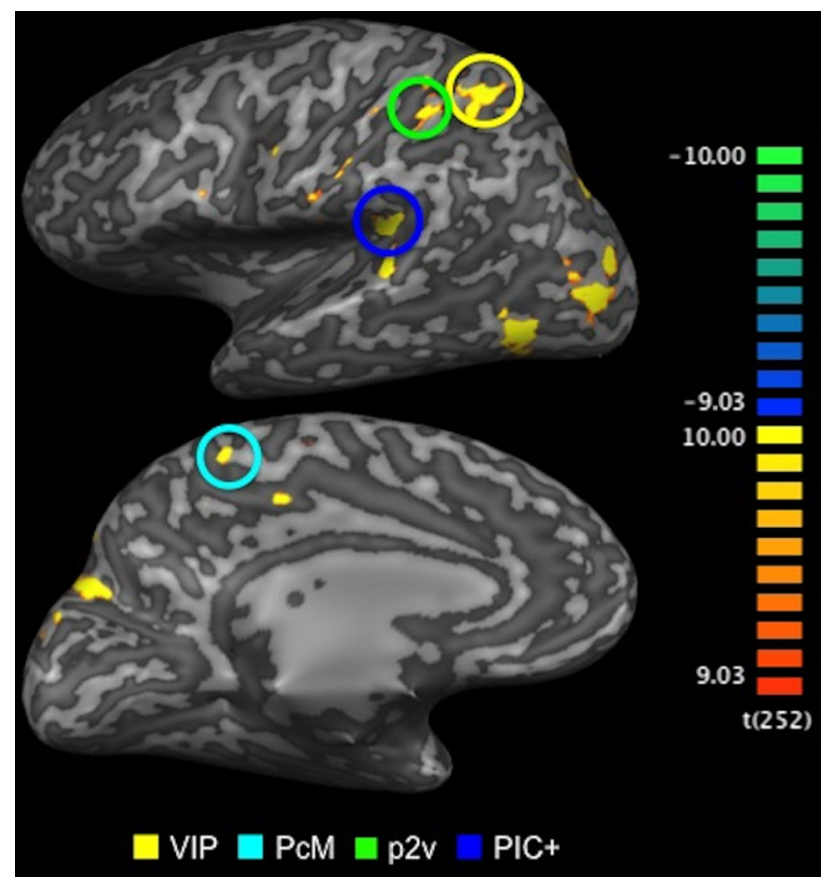

Fig. 6 Optic-flow selective areas localised using fMRI. Cortical areas that showed significantly greater BOLD responses to optic-flow stimulus than to random-motion stimulus ( $p<0.005$, uncorrected). Activation maps are superimposed on the inflated cortical surface of the left hemisphere in one representative subject (S1). Colour-coded bar (right panel) indicates statistical $t$ values (degree of freedom indicated in brackets). Four of the cortical areas selective for optic flow (VIP, PcM, p2v and PIC+) were successfully identified

the SIPS endpoints and the optic-flow selective areas (VIP, $\mathrm{PcM}, \mathrm{p} 2 \mathrm{v}$ and $\mathrm{PIC}+$ ).

Figure 7a depicts the relative position of SIPS with respect to the cortical areas selective for optic flow, in the left hemisphere of one representative subject ( $\mathrm{S} 1$; see Supplementary Figure 6 for other examples). PIC + is located in the posterior end of the lateral sulcus, near the ventro-lateral endpoints of SIPS. Three of the optic-flow selective areas (VIP, p2v and PcM) in the parietal cortex are located near the dorso-medial endpoints of SIPS. Whilst the ventro-lateral endpoints are observed in the vicinity of PIC + in a consistent manner across hemispheres, there are some degrees of variability in the spatial proximity between the superior parietal ROIs and dorsomedial endpoints of SIPS across hemispheres (Supplementary Figure 6). This variability may be due to the limitation of tractography in identifying the exact tract endpoints near smaller cortical regions located in the gyrus walls (Reveley et al. 2015). Figure 7b summarises the proportion of grey matter voxels in each optic-flow selective area located near the SIPS endpoints (see "Materials and methods"). Approximately 40 and $80 \%$ of voxels in each grey matter ROI are located in the vicinity of the SIPS endpoints, depending on the distance threshold for defining the spatial proximity between the grey matter voxels and tract endpoints (i.e. proximity was thresholded at 3 or $4.5 \mathrm{~mm}$ ). It seems highly likely that SIPS is part of the anatomical connection between the optic-flow selective areas in the superior parietal cortex (VIP, p2v and PcM) and the PIC+.

\section{Discussion}

Stratum proprium of interparietal sulcus (SIPS) was originally discovered in a post-mortem fibre dissection study by Sachs (1892) and was reproduced in another postmortem study by Vergani et al. (2014). Here, SIPS was successfully identified in the living human brain, using dMRI and tractography.

\section{Comparison of SIPS results with anatomical studies}

In this study, we investigated a white matter tract that has been largely overlooked in the visual and cognitive neuroscience, SIPS, using dMRI-based tractography and fascicle evaluation techniques. In spite of the challenges of using tractography to study little-investigated white matter tracts, SIPS was consistently identified across subjects and datasets. Between our dMRI results and the findings of the following anatomical studies, there is converging evidence supporting the existence of SIPS.

\section{Human post-mortem fibre dissection studies}

Most importantly, our results are consistent with human post-mortem fibre dissection studies (Fig. 3; Sachs 1892; Vergani et al. 2014). In those studies, SIPS was found to be located immediately posterior to the central sulcus, wrapping around the intraparietal sulcus, and to range between the superior parietal cortex and the lateral fissure. The position and the trajectory of SIPS reported in the postmortem fibre dissection studies are consistent with those of SIPS identified in vivo in three independent dMRI datasets in this study (Figs. 1, 2; Supplementary Figures 1-5).

To our knowledge, the first description of SIPS appeared in the atlas by Heinrich Sachs (1892); a German neurologist and neuroanatomist who studied under Wernicke. Sachs's atlas (1892) describes the white matter tracts in the post-mortem human brain in great detail, including the U-fibre system which has not been studied extensively in the living human brain. One of the short association tracts described is a tract termed stratum proprium fissurae interparietalis. Despite its relevance to perceptual and cognitive neuroscience, Sachs's atlas has been largely overlooked in the literature partly due to the lack of translation of the atlas from German to English (see Forkel 
Fig. 7 Spatial proximity between SIPS endpoints and cortical ROIs selective for optic-flow stimulation.

a Position of SIPS (magenta lines) in relation to the cortical ROIs identified by optic-flow stimulation using fMRI (dark blue PIC+; light blue PcM; yellow VIP; green $\mathrm{p} 2 \mathrm{v}$ ) in the left hemisphere in one representative subject (S1). b SIPS map coverage across all hemispheres. Vertical axis represents the proportion of voxels in each ROI within $3 \mathrm{~mm}$ (blue) and $4.5 \mathrm{~mm}$ (green) from SIPS endpoints. Error bars indicate \pm 1 SEM across hemispheres
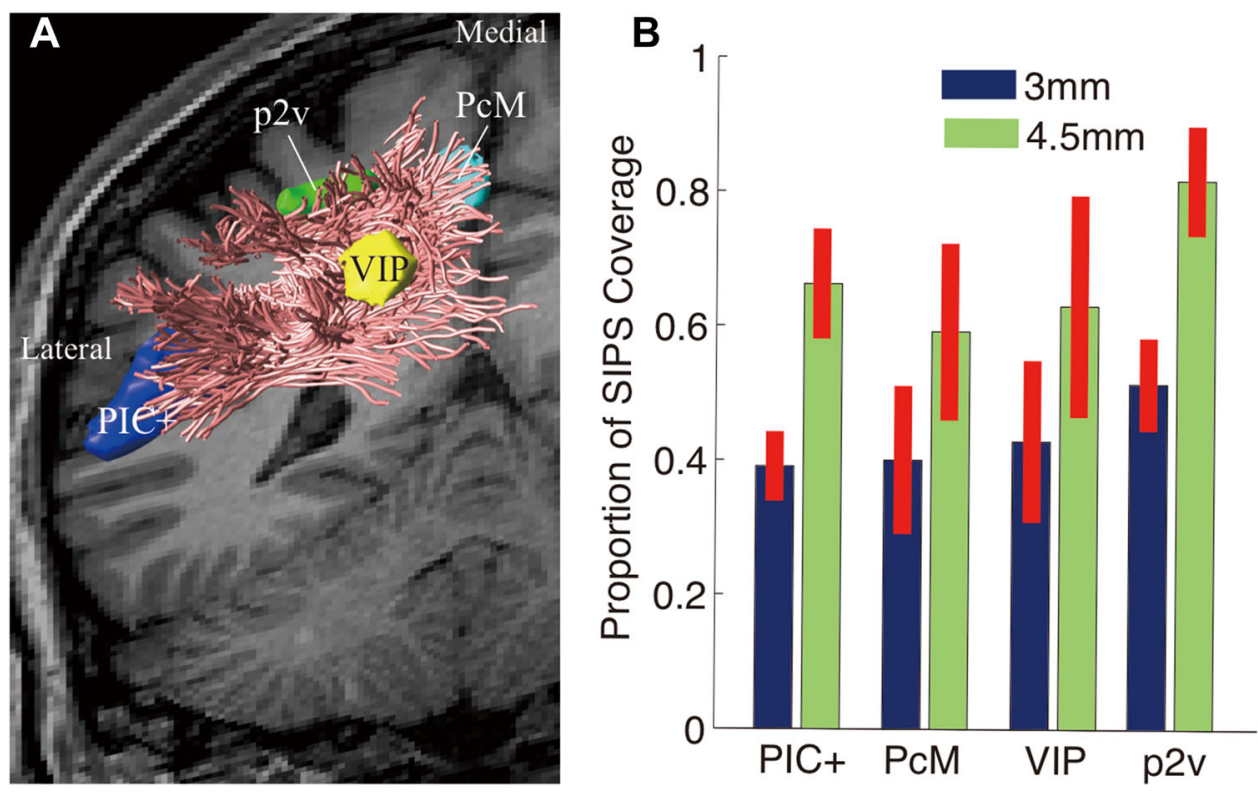

et al. 2015; for a historical review and English translation of the atlas). SIPS documented in this classical atlas was recently reproduced in a modern fibre dissection study by Vergani et al. (2014).

Our results describe the characteristics of SIPS identified in the living human brain, using modern neuroimaging techniques, which are highly consistent with the findings of the human post-mortem studies; hence providing further evidence for SIPS.

\section{Macaque tracer study}

Additionally, we note that a tract similar to human SIPS in the macaque brain has been reported in a tracer study. In their extensive study, Schmahmann and Pandya (2006) injected retrograde tracers into the macaque brain, and inspected the trajectory of white matter tracts from the injection sites. They reported several major white matter tracts seemingly homologous to human major white matter tracts identified in dissection studies (such as the inferior longitudinal fasciculus, and the superior longitudinal fasciculus); and those findings were later substantiated by macaque dMRI results (Schmahmann et al. 2007). In addition to the major white matter tracts, Schmahmann and Pandya (2006) also reported a fibre bundle wrapping around the intraparietal sulcus (Fig. 8). They note (page 120):

A dorsal fibre bundle lies subjacent to the cortex of the lower bank of the IPS and terminates in a columnar manner in area POa and in area IPd (Scs. 105, 113). These fibres continue medially and then curve around the depth of the IPS to ascend in the white matter of the superior parietal lobule. They terminate in area $I$ in a columnar manner and then first layers of area $3 \mathrm{~b}$ and $3 \mathrm{a}$ in the caudal bank and depth of the central sulcus (Sc. 105). Further caudally, these medially directed fibres terminate in a columnar manner in area 2 (Sc. 113).

Because of the compelling similarity between this fibre bundle identified in the macaque brain and human SIPS in terms of their anatomical positions and shapes, it could be hypothesised that this fibre bundle in macaque may be the homologue of human SIPS identified in this study.

Whilst the white matter structure of the macaque brain may be different from that of the human brain to some extent (Rilling et al. 2008), the fact that there is a white matter tract in the macaque brain that largely resembles human SIPS is encouraging for future investigations on human-macaque homology with respect to SIPS. There is a growing trend in neuroanatomy to use dMRI methods to compare the macro-scale white matter anatomy of the human brain and that of the macaque brain (Schmahmann et al. 2007; Oishi et al. 2011; Thiebaut de Schotten et al. 2011; Jbabdi et al. 2013; Mars et al. 2016; Takemura et al. 2017), which complements studies that investigate humanmacaque homology of cortical maps using fMRI (Brewer et al. 2002; Tsao et al. 2003, 2008; Wade et al. 2008; Goda et al. 2014; Kolster et al. 2014). It will be beneficial to study the precise anatomy of SIPS both in humans and macaques, to integrate the insights from macaque electrophysiology as well as tracer studies (Thiebaut de Schotten et al. 2012), and human fMRI studies investigating the neuronal network for multisensory integration guiding selfmotion perception. 


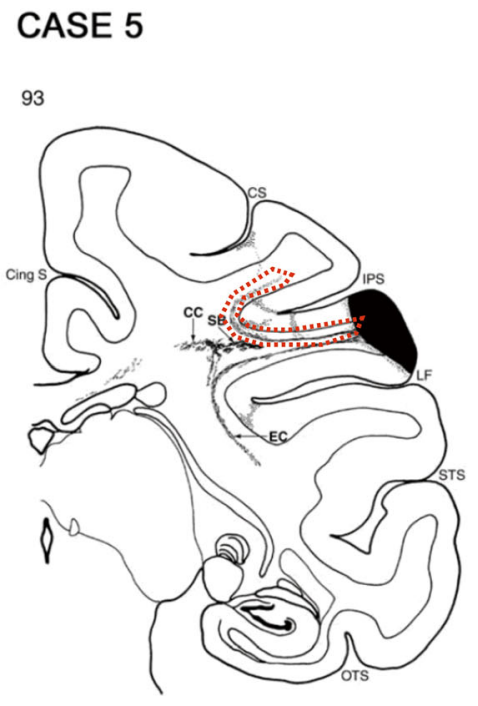

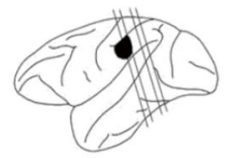
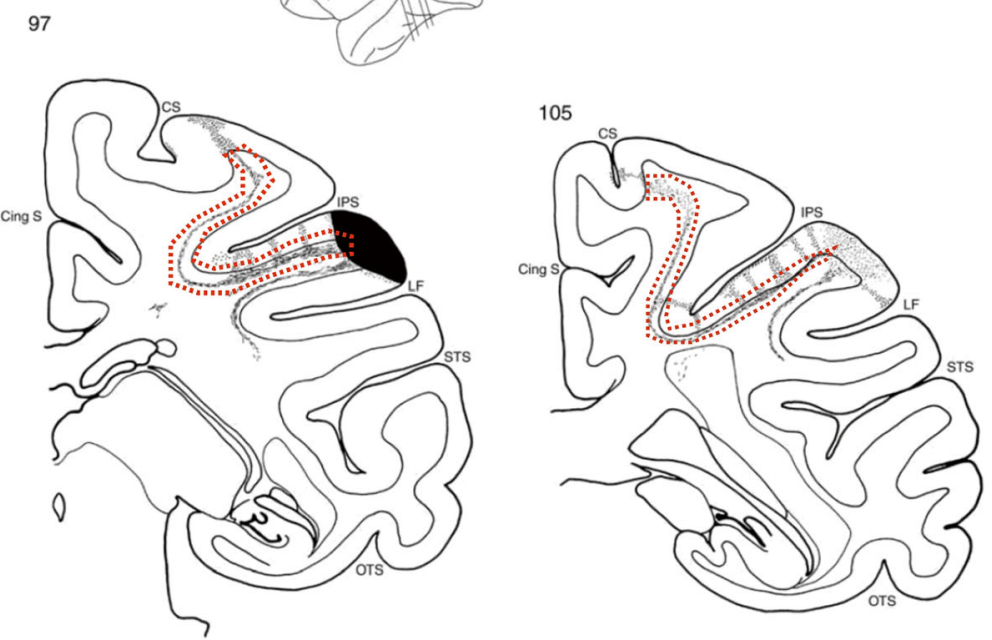

Fig. 8 A fibre bundle wrapping around the intraparietal sulcus reported in a macaque tracer study. This figure is reproduced from page 124 of Schmahmann and Pandya (2006, by permission of Oxford University Press, USA; http://www.oup.com). Each panel represents a coronal slice in the macaque brain (left panel anterior

\section{Functional localisation of optic-flow selective sensory regions}

Recent fMRI studies have shown that the sensory areas in the superior parietal regions (VIP, PcM, p2v) as well as an area around the posterior end of the lateral sulcus and parietal operculum (PIC+) are activated by optic-flow stimulation (Wall and Smith 2008; Cardin and Smith 2011; Greenlee et al. 2016). As in Uesaki and Ashida (2015), this study employed the functional localiser based on that described in Pitzalis et al. (2010), to identify VIP, PcM, $\mathrm{p} 2 \mathrm{v}$ and PIC + . The locations of those regions are consistent with those of the counterparts reported in previous studies (Cardin and Smith 2011; Uesaki and Ashida 2015).

We note that the definition and terminology of the area referred as PIC + in this study have been debated in the literature. In some earlier publications (Wall and Smith 2008; Cardin and Smith 2010, 2011; Uesaki and Ashida 2015), an area identified using optic-flow localisers was referred to as the parieto-insular vestibular cortex (PIVC) and was considered to be involved in integrating visual and vestibular information to guide self-motion perception. However, a recent vestibular fMRI study showed that PIVC is selectively responsive to vestibular stimulation, and is unlikely to be activated by visual stimulation (Frank et al. 2016; Greenlee et al. 2016). Frank et al. (2014) also suggested that PIVC and the area activated by visual stimulation, which is referred to as "PIC" in their study, are two independent areas. Their findings show that PIVC is purely vestibular, whilst PIC is predominantly visual but slice; right panel posterior slice). Cortical area marked in black is the injection site of anterograde tracer. Areas highlighted with dotted red lines in the white and grey matters indicate the axonal connections from the injection site, which defines a white matter tract wrapping around the intraparietal sulcus

also processes vestibular information. Here, we use "PIC+" to refer to the area around the posterior end of the lateral sulcus and parietal operculum, activated during optic-flow stimulation, as we did not examine the responsiveness of the area to vestibular stimuli.

Results suggest that the ventro-lateral endpoints of SIPS are near PIC+ (Fig. 7), but it is unclear whether these endpoints are also located near PIVC identified in vestibular fMRI studies (Frank and Greenlee 2014; Frank et al. 2014; Greenlee et al. 2016). Considering the proximity between PIC and PIVC (Frank and Greenlee 2014; Frank et al. 2014; Greenlee et al. 2016), it is possible that the ventro-lateral endpoints of SIPS are also adjacent to PIVC. Future studies should assess whether PIVC is directly connected to the superior part of the parietal cortex through SIPS, or indirectly connected via short-range connections with PIC + , to construct a more comprehensive model to understand how visual and vestibular signals are transmitted between these areas to guide self-motion perception.

\section{SIPS and its implication in multisensory integration}

Optic flow is a moving pattern on the retina caused by the relative motion between the observer and the scene, and is one of the most important visual cues to the estimation of self-motion (Gibson 1950, 1954; Warren and Hannon 1988). However, in most cases, perception of self-motion depends on integration of optic-flow information and signals from other sensory modalities such as the vestibular 
system. To understand the neuronal mechanism involved in the estimation of self-motion, it is important to elucidate how the visual and vestibular signals are integrated when we observe optic flow. Previous fMRI studies investigating the cortical areas selective for optic-flow and vestibular stimuli suggest that the sensory areas in the parietal cortex are involved in visuo-vestibular integration necessary for self-motion estimation (Wall and Smith 2008; Cardin and Smith 2011; Greenlee et al. 2016). Yet, the white matter anatomy that supports the communication amongst those areas has received very little attention in the literature of visual and cognitive neuroscience, even though the existence of SIPS has been known for over a century (Sachs 1892; Vergani et al. 2014).

One of the biggest advantages of the dMRI-based approach is that the positions of estimated white matter tracts and functionally localised cortical areas can be compared in the brain of the same individual. This is particularly important to hypothesise the types of information that are transferred via the tracts of interest (Kim et al. 2006; Greenberg et al. 2012; Yeatman et al. 2013; Takemura et al. 2016b; Rokem et al. 2017). We combined dMRI and fMRI, and analysed the spatial proximity between the SIPS endpoints and the optic-flow selective cortical areas localised within the same subjects. Results show that the dorso-medial SIPS endpoints are near VIP, PcM and p2v, and the ventro-lateral SIPS endpoints near $\mathrm{PIC}+$, despite some variability in the spatial proximity between the superior parietal ROIs and dorso-medial endpoints of SIPS across hemispheres (Fig. 7; Supplementary Figure 6). These cortical areas have been associated with the convergence of visual and vestibular information regarding self-motion (Fetsch et al. 2009; Prsa et al. 2012; Uesaki and Ashida 2015; Kleinschmidt et al. 2002; Kovács et al. 2008; Wiest et al. 2004; Butler et al. 2010). Our results and those findings together suggest that communication between VIP, PcM, p2v and PIC+ likely plays a crucial role in multisensory integration necessary for accurate perception of self-motion, and that it is supported by SIPS. The spatial relationship between SIPS and the optic-flow selective areas will have implications for interpreting the consequences of white matter lesions that include SIPS, or exploring the neuronal basis of individual differences in self-motion perception.

\section{Limitations and directions for future research}

Our findings show that SIPS is an important structure supporting communication amongst sensory areas in the parietal cortex. It must be noted, however, that it is possible that our results represent only a subset of SIPS. Our tractography results are based on in vivo dMRI data with $1.25-2 \mathrm{~mm}$ isotropic spatial resolution, but LiFE analysis generally supports larger portions of fibre tracts as the data resolution improves (Pestilli et al. 2014; Takemura et al. 2017). Tractography based on data with higher resolutions would likely allow for the extraction of a larger portion of SIPS. Likewise, estimation of cortical endpoints would be more accurate with data of better quality, as some cortical endpoints are still missed even with the best dMRI data currently attainable (Reveley et al. 2015).

Other limitations that should be considered include the lower $b$ value $\left(1000 \mathrm{~s} / \mathrm{mm}^{2}\right)$ used in the acquisition of KU dataset. It has been suggested that lower $b$ values are not optimal for resolving crossing fibres (Tournier et al. 2004; Alexander and Barker 2005), despite their relatively higher signal-to-noise ratio. To compensate for this limitation, we included HCP data acquired with higher $b$ values, higher spatial and angular resolution from a large number of subjects. Results demonstrated the compelling consistency in the tractography results across the three datasets. This approach complements the relative disadvantage of the current version of LiFE that it only accepts single-shell data. Contrary to the single-shell approach, multi-shell approaches can be used to generate alternative matrices, which provide additional information regarding tissue microstructures that cannot be captured at a voxel level using single-shell approaches.

It should also be noted that, although SIPS is discussed mainly within the contexts of multisensory integration and optic-flow processing in this article, the SIPS endpoints appear to be near the cortical areas involved in other cognitive functions such as attention (Corbetta and Shulman 2002; Yantis et al. 2002; Bisley and Goldberg 2003), memory (Cabesa et al. 2008; Koenigs et al. 2009; Uncapher and Wagner 2009; Chun and Johnson 2011), motor sequence learning (Rizzolatti and Luppino 2001), visuomotor control (Culham et al. 2006), decision making (Platt and Glimcher 1999), body-ownership (Blanke 2012) and social cognition (Decety and Lamm 2007). To further understand the implications of SIPS in relation to human behaviour, it may be useful to assess the relationship between individual differences in diffusion properties along SIPS (e.g. FA) and behavioural measures, as has been done for other white matter tracts in previous studies (Genç et al. 2011; Yeatman et al. 2012a; Tavor et al. 2014; Gomez et al. 2015; Leong et al. 2016).

On the other hand, for relatively small tracts like SIPS, especially if they cross or kiss other major tracts like AF (Fig. 3), it is difficult to interpret the results regarding diffusion properties derived from diffusion tensor-based analyses. Possible solutions to these limitations include acquiring dMRI data at a higher angular resolution with multiple $b$ values, use of other dMRI techniques (e.g. neurite orientation dispersion and density imaging; NODDI; Zhang et al. 2012), and combining dMRI data 
with quantitative MRI measurements, to evaluate the physical properties of SIPS in a manner relatively independent from fibre crossings (Dell'Acqua et al. 2013; Mezer et al. 2013; Yeatman et al. 2014b; Mohammadi et al. 2015; Stikov et al. 2015; Weiskopf et al. 2015). Future dMRI studies should examine the properties of SIPS in relation to cognitive functions, as well as development and diseases, with consideration to shortcomings of currently available MRI techniques. To facilitate further research on SIPS, we describe the methods and provide open-source implementations [https://github.com/htakemur/SIPS; https:// github.com/yeatmanlab/AFQ].

\section{Conclusion}

This study identified the white matter tract, SIPS, in the living human brain using dMRI and tractography. It is located immediately posterior to the central sulcus and around the intraparietal sulcus; and connects the superior and inferior parts of the parietal cortex. The location and the trajectory of SIPS are consistent with those observed in post-mortem fibre dissection studies by Sachs (1892) and Vergani et al. (2014). SIPS was identified consistently across a large number of subjects from three independent dMRI datasets, and the existence of the tract was further corroborated by statistical evidence. These findings place SIPS in a good position to channel neuronal communication between the distant cortical areas underlying visuovestibular integration necessary for optic-flow processing and perception of self-motion. In vivo identification and characterisation of SIPS using dMRI data and tractography will open new avenues to further studying this tract in relation to diseases, development and brain functions.

\footnotetext{
Acknowledgements This study was conducted using the MRI scanner and related facilities of Kokoro Research Center, Kyoto University and was supported by Japan Society for the Promotion of Science (JSPS) KAKENHI (B26285165 to HA; $17 \mathrm{H} 04684$ to HT), Grant-in-Aid for JSPS Fellows (to HT and MU) and JSPS Postdoctoral Fellowship for Research Abroad (HT). HT is a JSPS Superlative Postdoctoral Fellow. We thank Franco Pestilli for his comments on the earlier version of the manuscript, Daniel Bullock for discussions, Brian Wandell, Lee Michael Perry, Shumpei Ogawa and Ariel Rokem for their technical advice on the study, Michele Furlan for rendering analysis tools, Kaoru Amemiya for her support in analysis of the HCP dataset. We also thank Kaoru Amano, Atsushi Wada and Noboru Nushi for providing the computing environment to run the analyses. We also acknowledge the stimulated discussion in the meetings of the Cooperative Research Project of the Research Institute of Electrical Communication, Tohoku University. Data were provided in part by the Human Connectome Project, WU-Minn Consortium (Principal Investigators: David Van Essen and Kamil Ugurbil 1U54MH091657) funded by the $16 \mathrm{NIH}$ Institutes and Centres that support the NIH Blueprint for Neuroscience Research, and by the McDonnell Center for Systems Neuroscience at Washington University and in part by the Human Connectome Project, MGH-USC Consortium (Principal
}

Investigators: Bruce R. Rosen, Arthur W. Toga and Van Wedeen U01MH093765) funded by the NIH Blueprint Initiative for Neuroscience Research grant, the National Institutes of Health Grant P41EB015896, and the Instrumentation Grants S10RR023043, 1S10RR023401, 1S10RR019307.

Open Access This article is distributed under the terms of the Creative Commons Attribution 4.0 International License (http://crea tivecommons.org/licenses/by/4.0/), which permits unrestricted use, distribution, and reproduction in any medium, provided you give appropriate credit to the original author(s) and the source, provide a link to the Creative Commons license, and indicate if changes were made.

Author contributions Designed the study: MU HA. Performed the experiments: MU HA. Analysed the data: MU HT. Contributed analysis tools: HT. Wrote the paper: MU HT HA.

\section{References}

Alexander DC, Barker GJ (2005) Optimal imaging parameters for fiber-orientation estimation in diffusion MRI. Neuroimage 27(2):357-367

Basser PJ, Pierpaoli C (1996) Microstructural and physiological features of tissues elucidated by quantitative-diffusion-tensor MRI. J Magn Reson B 111:209-219

Biagi L, Crespi SA, Tosetti M, Morrone MC (2015) BOLD response selective to flow-motion in very young infants. PLoS Biol 13:e1002260

Bisley JW, Goldberg ME (2003) Neuronal activity in the lateral intraparietal area and spatial attention. Science 299(5603):81-86

Blanke O (2012) Multisensory brain mechanisms of bodily selfconsciousness. Nat Rev Neurosci 13:556-571

Brewer AA, Press WA, Logothetis NK, Wandell BA (2002) Visual areas in macaque cortex measured using functional magnetic resonance imaging. J Neurosci 22:10416-10426

Bullock TH, Bennett MV, Johnston D, Josephson R, Marder E, Fields RD (2005) The neuron doctrine, redux. Science 310(5749):791-793

Bürgel U, Amunts K, Hoemke L, Mohlberg H, Gilsbach JM, Zilles K (2006) White matter fiber tracts of the human brain: threedimensional mapping at microscopic resolution, topography and intersubject variability. Neuroimage 29(4):1092-1105

Butler JS, Smith ST, Campos JL, Bülthoff HH (2010) Bayesian integration of visual and vestibular signals for heading. $\mathrm{J}$ Vis $10: 23$

Cabeza R, Ciaramelli E, Olson IR, Moscovitch M (2008) The parietal cortex and episodic memory: an attentional account. Nat Revi Neurosci 9(8):613-625

Caiafa CF, Pestilli F (2017) Multidimensional encoding of brain connectomes. bioRxiv: 107607

Cardin V, Smith AT (2010) Sensitivity of human visual and vestibular cortical regions to egomotion-compatible visual stimulation. Cereb Cortex 20:1964-1973

Cardin V, Smith AT (2011) Sensitivity of human visual cortical area V6 to stereoscopic depth gradients associated with self-motion. J Neurophysiol 106:1240-1249

Catani M, Ffytche DH (2005) The rises and falls of disconnection syndromes. Brain 128:2224-2239

Catani M, Thiebaut de Schotten M (2012) Atlas of human brain connections. Oxford University Press, Oxford

Catani M, Howard RJ, Pajevic S, Jones DK (2002) Virtual in vivo interactive dissection of white matter fasciculi in the human brain. Neuroimage 17:77-94 
Catani M, Jones DK, Ffytche DH (2005) Perisylvian language networks of the human brain. Ann Neurol 57:8-16

Catani M, Allin MP, Husain M, Pugliese L, Mesulam MM, Murray RM, Jones DK (2007) Symmetries in human brain language pathways correlate with verbal recall. Proc Natl Acad Sci USA 104(43):17163-17168

Catani M, Mesulam MM, Jakobsen E, Malik F, Martersteck A, Wieneke C, Thompson CK, Thiebaut de Schotten M, Dell'Acqua F, Weintraub S, Rogalski E (2013) A novel frontal pathway underlies verbal fluency in primary progressive aphasia. Brain 136:2619-2628

Chun MM, Johnson MK (2011) Memory: enduring traces of perceptual and reflective attention. Neuron 72(4):520-535

Corbetta M, Shulman GL (2002) Control of goal-directed and stimulus-driven attention in the brain. Nat Rev Neurosci 3:201-215

Craddock RC, Jbabdi S, Yan CG, Vogelstein JT, Castellanos FX, Di Martino A, Kelly C, Heberlein K, Colcombe S, Milham MP (2013) Imaging human connectomes at the macroscale. Nat Methods 10:524-539

Culham JC, Cavina-Pratesi CC, Singhal A (2006) The role of parietal cortex in visuomotor control: what have we learned from neuroimaging? Neuropsychologia 44:2668-2684

Decety J, Lamm C (2007) The role of the right temporoparietal junction in social interaction: how low-level computational processes contribute to meta-cognition. Neuroscientist 13(6):580-593

Dell'Acqua F, Simmons A, Williams SC, Catani M (2013) Can spherical deconvolution provide more information than fiber orientations? Hindrance modulated orientational anisotropy, a true-tract specific index to characterize white matter diffusion. Hum Brain Mapp 34(10):2464-2483

Desikan RS, Ségonne F, Fischl B, Quinn BT, Dickerson BC, Blacker D, Buckner RL, Dale AM, Maguire RP, Hyman BT, Albert MS (2006) An automated labeling system for subdividing the human cerebral cortex on MRI scans into gyral based regions of interest. Neuroimage 31(3):968-980

Deutschländer A, Bense S, Stephan T, Schwaiger M, Dieterich M, Brandt T (2004) Rollvection versus linearvection: comparison of brain activations in PET. Hum Brain Mapp 21:143-153

Duan Y, Norcia AM, Yeatman JD, Mezer A (2015) The structural properties of major white matter tracts in strabismic amblyopia. Invest Ophthalmol Vis Sci 56:5152-5160

Fan Q, Nummenmaa A, Witzel T, Zanzonico R, Keil B, Cauley S, Polimeni JR, Tisdall D, Van Dijk KR, Buckner RL, Wedeen VJ, Rosen BR, Wald LL (2014) Investigating the capability to resolve complex white matter structures with high $b$-value diffusion magnetic resonance imaging on the MGH-USC connectome scanner. Brain Connect 4(9):718-726

Fan Q, Witzel T, Nummenmaa A, Van Dijk KR, Van Horn JD, Drews MK, Somerville LH, Sheridan MA, Santillana RM, Snyder J, Hedden T, Shaw EE, Hollinshead MO, Renvall V, Zanzonico R, Keil B, Cauley S, Polimeni JR, Tisdall D, Buckner RL, Wedeen VJ, Wald LL, Toga AW, Rosen BR (2016) MGH-USC Human Connectome Project datasets with ultra-high $b$-value diffusion MRI. Neuroimage 124(Pt B):1108-1114

Fetsch CR, Turner AH, DeAngelis GC, Angelaki DE (2009) Dynamic reweighting of visual and vestibular cues during self-motion perception. J Neurosci 29:15601-15612

Fields RD (2008) White matter in learning, cognition and psychiatric disorders. Trends Neurosci 31(7):361-370

Fields RD (2015) A new mechanism of nervous system plasticity: activity-dependent myelination. Nat Rev Neurosci 16(12):756-767

Fischl B (2012) FreeSurfer. Neuroimage 62:774-781

Forkel SJ, Mahmood S, Vergani F, Catani M (2015) The white matter of the human cerebrum: part I. The occipital lobe by Heinrich Sachs. Cortex 62:182-202
Frank LR (2001) Anisotropy in high angular resolution diffusionweighted MRI. Magn Reson Med 45:935-939

Frank SM, Greenlee MW (2014) An MRI-compatible caloric stimulation device for the investigation of human vestibular cortex. J Neurosci Methods 235:208-218

Frank SM, Baumann O, Mattingley JB, Greenlee MW (2014) Vestibular and visual responses in human posterior insular cortex. J Neurophysiol 112:2481-2491

Frank SM, Wirth AM, Greenlee MW (2016) Visual-vestibular processing in the human sylvian fissure. $\mathrm{J}$ Neurophysiol 116:263-271

Friston KJ, Ashburner J (2004) Generative and recognition models for neuroanatomy. Neuroimage 23:21-24

Genç E, Bergmann J, Tong F, Blake R, Singer W, Kohler A (2011) Callosal connections of primary visual cortex predict the spatial spreading of binocular rivalry across the visual hemifields. Front Hum Neurosci 5:161. doi:10.3389/fnhum.2011.00161

Gibson JJ (1950) The perception of the visual world. Houghton Mifflin, Boston

Gibson JJ (1954) The visual perception of objective motion and subjective movement. Psychol Rev 61:304-314

Goda N, Tachibana A, Okazawa G, Komatsu H (2014) Representation of the material properties of objects in the visual cortex of nonhuman primates. J Neurosci 34:2660-2673

Gomez J, Pestilli F, Witthoft N, Golarai G, Liberman A, Poltoratski S, Yoon J, Grill-Spector K (2015) Functionally defined white matter reveals segregated pathways in human ventral temporal cortex associated with category-specific processing. Neuron 85:216-227

Greenberg AS, Verstynen T, Chiu YC, Yantis S, Schneider W, Behrmann M (2012) Visuotopic cortical connectivity underlying attention revealed with white-matter tractography. J Neurosci 32:2773-2782

Greenlee MW, Frank SM, Kaliuzhna M, Blanke O, Bremmer F, Churan J, Cuturi LF, MacNeilage PR, Smith AT (2016) Multisensory integration in self motion perception. Multisens Res 29:525-556

Guevara M, Román C, Houenou J, Duclap D, Poupon C, Mangin JF, Guevara P (2017) Reproducibility of superficial white matter tracts using diffusion-weighted imaging tractography. Neuroimage 147:703-725

Honey CJ, Sporns O (2008) Dynamical consequences of lesions in cortical networks. Hum Brain Mapp 29:802-809

Jbabdi S, Lehman JF, Haber SN, Behrens TE (2013) Human and monkey ventral prefrontal fibers use the same organizational principles to reach their targets: tracing versus tractography. J Neurosci 33:3190-3201

Jeurissen B, Tournier JD, Dhollander T, Connelly A, Sijbers J (2014) Multi-tissue constrained spherical deconvolution for improved analysis of multi-shell diffusion MRI data. NeuroImage 103:411-426

Kemerdere R, de Champfleur NM, Deverdun J, Cochereau J, MoritzGasser S, Herbet G, Duffau H (2016) Role of the left frontal aslant tract in stuttering: a brain stimulation and tractographic study. J Neurol 263(1):157-167

Kim M, Ducros M, Carlson T, Ronen I, He S, Ugurbil K, Kim D-S (2006) Anatomical correlates of the functional organization in the human occipitotemporal cortex. Magn Reson Imaging 24:583-590

Kinoshita M, de Champfleur NM, Deverdun J, Moritz-Gasser S, Herbet G, Duffau H (2015) Role of fronto-striatal tract and frontal aslant tract in movement and speech: an axonal mapping study. Brain Struct Funct 220(6):3399-3412

Kleinschmidt A, Thilo KV, Büchel C, Gresty MA, Bronstein AM, Frackowiak RSJ (2002) Neural correlates of visual-motion perception as object- or self-motion. Neuroimage 16:873-882 
Koenigs M, Barbey AK, Postle BR, Grafman J (2009) Superior parietal cortex is critical for the manipulation of information in working memory. J Neurosci 29(47):14980-14986

Kolster H, Janssens T, Orban GA, Vanduffel W (2014) The retinotopic organization of macaque occipitotemporal cortex anterior to V4 and caudoventral to the middle temporal (MT) cluster. J Neurosci 34:10168-10191

Kovács G, Raabe M, Greenlee MW (2008) Neural correlates of visually induced self-motion illusion in depth. Cereb Cortex 18:1779-1787

Kronfeld-Duenias V, Amir O, Ezrati-Vinacour R, Civier O, BenShachar M (2016) The frontal aslant tract underlies speech fluency in persistent developmental stuttering. Brain Struct Funct 221(1):365-381

Lebel C, Gee M, Camicioli R, Wieler M, Martin W, Beaulieu C (2012) Diffusion tensor imaging of white matter tract evolution over the lifespan. Neuroimage 60:340-352

Lee Masson H, Wallraven C, Petit L (2017) "Can touch this": crossmodal shape categorization performance is associated with microstructural characteristics of white matter association pathways. Hum Brain Mapp 38(2):842-854

Leong JK, Pestilli F, Wu CC, Samanez-Larkin GR, Knutson B (2016) White-matter tract connecting anterior insula to nucleus accumbens correlates with reduced preference for positively skewed gambles. Neuron 89:63-69

Malania M, Konrad J, Jägle H, Werner JS, Greenlee MW (2017) Compromised integrity of central visual pathways in patients with macular degeneration. Invest Ophthalmol Vis Sci 58:2939-2947

Mars RB, Foxley S, Verhagen L, Jbabdi S, Sallet J, Noonan MP, Neubert F-X, Andersson JL, Croxson PL, Dunbar RIM, Khrapitchev AA, Sibson NR, Miller KL, Rushworth MFS (2016) The extreme capsule fiber complex in humans and macaque monkeys: a comparative diffusion MRI tractography study. Brain Struct Funct 221(8):4059-4071

Mezer A, Yeatman JD, Stikov N, Kay KN, Cho NJ, Dougherty RF, Perry ML, Parvizi J, Hua LH, Butts-Pauly K, Wandell BA (2013) Quantifying the local tissue volume and composition in individual brains with magnetic resonance imaging. Nat Med 19(12):1667-1672

Mohammadi S, Carey D, Dick F, Diedrichsen J, Sereno MI, Reisert M, Callaghan MF, Weiskopf N (2015) Whole-brain in vivo measurements of the axonal $g$-ratio in a group of 37 healthy volunteers. Front Neurosci 9:441. doi:10.3389/fnins.2015.00441

Mori S, Zhang J (2006) Principles of diffusion tensor imaging and its applications to basic neuroscience research. Neuron 51:527-539

Ogawa S, Takemura H, Horiguchi H, Terao M, Haji T, Pestilli F, Yeatman JD, Tsuneoka H, Wandell BA, Masuda Y (2014) White matter consequences of retinal receptor and ganglion cell damage. Invest Ophthalmol Vis Sci 55:6976-6986

Oishi K, Zilles K, Amunts K, Faria A, Jiang H, Li X, Akhter K, Hua K, Woods R, Toga AW, Bruce Pike G, Rosa-Neto P, Evans A, Zhang J, Huang H, Miller MI, van Zijl PCM, Mazziotta J, Mori S (2008) Human brain white matter atlas: identification and assignment of common anatomical structures in superficial white matter. Neuroimage 43:447-457

Oishi K, Huang H, Yoshioka T, Ying SH, Zee DS, Zilles K, Amunts K, Woods R, Toga AT, Pike GB, Rosa-Neto P, Evans AC, van Zijl PCM, Mazziotta JC, Mori S (2011) Superficially located white matter structures commonly seen in the human and the macaque brain with diffusion tensor imaging. Brain Connect $1: 37-47$

Pajevic S, Pierpaoli C (1999) Color schemes to represent the orientation of anisotropic tissues from diffusion tensor data: application to white matter fiber tract mapping in the human brain. Magn Reson Med 42:526-540
Pestilli F, Yeatman JD, Rokem A, Kay KN, Wandell BA (2014) Evaluation and statistical inference for human connectomes. Nat Methods 11:1058-1063

Pitzalis S, Sereno MI, Committeri G, Fattori P, Galati G, Patria F, Galletti C (2010) Human v6: the medial motion area. Cereb Cortex 20:411-424

Platt ML, Glimcher PW (1999) Neural correlates of decision variables in parietal cortex. Nature 400(6741):233-238

Prsa M, Gale S, Blanke O (2012) Self-motion leads to mandatory cue fusion across sensory modalities. J Neurophysiol 108:2282-2291

Prsa M, Jimenez-Rezende D, Blanke O (2015) Inference of perceptual priors from path dynamics of passive self-motion. J Neurophysiol 113:1400-1413

Reveley C, Seth AK, Pierpaoli C, Silva AC, Yu D, Saunders RC, Leopold DA, Ye FQ (2015) Superficial white matter fiber systems impede detection of long-range cortical connections in diffusion MR tractography. Proc Natl Acad Sci USA. 112:E2820-E2828

Rilling JK, Glasser MF, Preuss TM, Ma X, Zhao T, Hu X, Behrens TEJ (2008) The evolution of the arcuate fasciculus revealed with comparative DTI. Nat Neurosci 11:426-428

Rizzolatti G, Luppino G (2001) The cortical motor system. Neuron 31(6):889-901

Rohde GK, Barnett AS, Basser PJ, Marenco S, Pierpaoli C (2004) Comprehensive approach for correction of motion and distortion in diffusion-weighted MRI. Magn Reson Med 51:103-114

Rokem A, Yeatman JD, Pestilli F, Kay KN, Mezer A, van der Walt S, Wandell BA (2015) Evaluating the accuracy of diffusion MRI models in white matter. PLoS ONE 10:e0123272

Rokem A, Takemura H, Bock A, Scherf KS, Behrmann M, Wandell B, Fine I, Bridge H, Pestilli F (2017) The visual white matter: The application of diffusion MRI and fiber tractography to vision science. J Vis 17(2):4

Sachs H (1892) Das hemisphärenmark des menschlichen grosshirns. Verlag von georg thieme, Leipzig

Schmahmann JD, Pandya D (2006) Fiber pathways of the brain. Oxford University Press, New York

Schmahmann JD, Pandya DN, Wang R, Dai G, D'Arceuil HE, de Crespigny AJ, Wedeen VJ (2007) Association fibre pathways of the brain: parallel observations from diffusion spectrum imaging and autoradiography. Brain 130:630-653

Smith RE, Tournier JD, Calamante F, Connelly A (2012a) Anatomically-constrained tractography: improved diffusion MRI streamlines tractography through effective use of anatomical information. Neuroimage 62(3):1924-1938

Smith AT, Wall MB, Thilo KV (2012b) Vestibular inputs to human motion-sensitive visual cortex. Cereb Cortex 22:1068-1077

Smith AT, Beer AL, Furlan M, Mars RB (2017) Connectivity of the cingulate sulcus visual area $(\mathrm{CSv})$ in the human cerebral cortex. Cereb Cortex. doi:10.1093/cercor/bhx002

Sotiropoulos SN, Jbabdi S, Xu J, Andersson JL, Moeller S, Auerbach EJ, Glasser MF, Hernandez M, Sapiro G, Jenkinson M, Feinberg DA, Yacoub E, Lenglet C, Van Essen DC, Ugurbil K, Behrens TE, Consortium, W. U-Minn HCP (2013) Advances in diffusion MRI acquisition and processing in the Human Connectome Project. Neuroimage. 80:125-143

Stikov N, Campbell JS, Stroh T, Lavelée M, Frey S, Novek J, Naura S, Ho MK, Bedell BJ, Dougherty RF, Leppert IR, Boudreau M, Narayanan S, Duval T, Cohen-Adad J, Picard PA, Gasecka A, Cote D, Bruce Pike G (2015) In vivo histology of the myelin g-ratio with magnetic resonance imaging. Neuroimage 118:397-405

Takemura H, Caiafa CF, Wandell BA, Pestilli F (2016a) Ensemble tractography. PLoS Comput Biol 12:e1004692

Takemura H, Rokem A, Winawer J, Yeatman JD, Wandell BA, Pestilli F (2016b) A major human white-matter pathway between dorsal and ventral visual cortex. Cereb Cortex 26:2205-2214 
Takemura H, Pestilli F, Weiner KS, Keliris GA, Landi SM, Sliwa J, Ye FQ, Barnett MA, Leopold DA, Freiwald WA, Logothetis NK, Wandell BA (2017) Occipital white matter tracts in human and macaque. Cereb Cortex 27:3346-3359

Tavor I, Yablonski M, Mezer A, Rom S, Assaf Y, Yovel G (2014) Separate parts of occipito-temporal white matter fibers are associated with recognition of faces and places. Neuroimage 86:123-130

Thiebaut de Schotten M, Kinkingnehun S, Delmaire C, Lehericy S, Duffau H, Thivard L, Volle E, Levy R, Dubois B, Bartolomeo P (2008) Visualization of disconnection syndromes in humans. Cortex 44(8):1097-1103

Thiebaut de Schotten M, Dell'Acqua F, Forkel SJ, Simmons A, Vergani F, Murphy DG, Catani M (2011) A lateralized brain network for visuospatial attention. Nat Neurosci 14:1245-1246

Thiebaut de Schotten M, Dell'Acqua F, Valabregue R, Catani M (2012) Monkey to human comparative anatomy of the frontal lobe association tracts. Cortex 48(1):82-96

Tournier JD, Calamante F, Gadian DG, Connelly A (2004) Direct estimation of the fiber orientation density function from diffusion-weighted MRI data using spherical deconvolution. NeuroImage 23(3):1176-1185

Tournier JD, Calamante F, Connelly A (2007) Robust determination of the fibre orientation distribution in diffusion MRI: nonnegativity constrained super-resolved spherical deconvolution. Neuroimage 35:1459-1472

Tournier JD, Calamante F, Connelly A (2012) MRtrix: diffusion tractography in crossing fiber regions. Int $\mathrm{J}$ Imaging Syst Technol 22:53-66

Tsao DY, Vanduffel W, Sasaki Y, Fize D, Knutsen TA, Mandeville JB, Wald LL, Dale AM, Rosen BR, Van Essen DC, Livingstone MS, Orban GA, Tootell RB (2003) Stereopsis activates V3A and caudal intraparietal areas in macaques and humans. Neuron 39:555-568

Tsao DY, Moeller S, Freiwald WA (2008) Comparing face patch systems in macaques and humans. Proc Natl Acad Sci USA 105:19514-19519

Uesaki M, Ashida H (2015) Optic-flow selective cortical sensory regions associated with self-reported states of vection. Front Psychol 6:775

Uncapher MR, Wagner AD (2009) Posterior parietal cortex and episodic encoding: insights from fMRI subsequent memory effects and dual-attention theory. Neurobiol Learn Mem 91:139-154

Van Essen DC, Smith SM, Barch DM, Behrens TE, Yacoub E, Ugurbil K, Consortium, W. U-Minn HCP (2013) The WU-Minn Human Connectome Project: an overview. Neuroimage 80:62-79

Vergani F, Mahmood S, Morris CM, Mitchell P, Forkel SJ (2014) Intralobar fibres of the occipital lobe: a post mortem dissection study. Cortex 56:145-156

Wada A, Sakano Y, Ando H (2016) Differential responses to a visual self-motion signal in human medial cortical regions revealed by wide-view stimulation. Front Psychol 7:309

Wade A, Augath M, Logothetis N, Wandell B (2008) fMRI measurements of color in macaque and human. J Vis 8(6):1-19

Wakana S, Jiang H, Nagae-Poetscher LM, van Zijl PC, Mori S (2004) Fiber tract-based atlas of human white matter anatomy. Radiology 230:77-87
Wall MB, Smith AT (2008) The representation of egomotion in the human brain. Curr Biol 18:191-194

Wandell BA (2016) Clarifying human white matter. Annu Rev Neurosci 39:103-128

Wandell BA, Yeatman JD (2013) Biological development of reading circuits. Curr Opin Neurobiol 23(2):261-268

Warren WH Jr, Hannon DJ (1988) Direction of self-motion is perceived from optical flow. Nature 336:162-163

Wedeen VJ, Rosene DL, Wang R, Dai G, Mortazavi F, Hagmann P, Kaas JH, Tseng WY (2012) The geometric structure of the brain fiber pathways. Science 335:1628-1634

Weiner KS, Yeatman JD, Wandell BA (2016) The posterior arcuate fasciculus and the vertical occipital fasciculus. Cortex (epub ahead of print)

Weiskopf N, Mohammadi S, Lutti A, Callaghan MF (2015) Advances in MRI-based computational neuroanatomy: from morphometry to in vivo histology. Curr Opin Neurol 28(4):313-322

Wiest G, Zimprich F, Prayer D, Czech T, Serles W, Baumgartner C (2004) Vestibular processing in human paramedian precuneus as shown by electrical cortical stimulation. Neurology 62:473-475

Yantis S, Schwarzbach J, Serences JT, Carlson RL, Steinmetz MA, Pekar JJ, Courtney SM (2002) Transient neural activity in human parietal cortex during spatial attention shifts. Nat Neurosci 5(10):995-1002

Yeatman JD, Dougherty RF, Rykhlevskaia E, Sherbondy AJ, Deutsch GK, Wandell BA, Ben-Shachar M (2011) Anatomical properties of the arcuate fasciculus predict phonological and reading skills in children. J Cogn Neurosci 23:3304-3317

Yeatman JD, Dougherty RF, Ben-Shachar M, Wandell BA (2012a) Development of white matter and reading skills. Proc Natl Acad Sci USA 109:E3045-E3053

Yeatman JD, Dougherty RF, Myall NJ, Wandell BA, Feldman HM (2012b) Tract profiles of white matter properties: automating fiber-tract quantification. PLoS ONE 7:e49790

Yeatman JD, Rauschecker AM, Wandell BA (2013) Anatomy of the visual word form area: adjacent cortical circuits and long-range white matter connections. Brain Lang 125:146-155

Yeatman JD, Wandell BA, Mezer AA (2014a) Lifespan maturation and degeneration of human brain white matter. Nat Commun $5: 4932$

Yeatman JD, Weiner KS, Pestilli F, Rokem A, Mezer A, Wandell BA (2014b) The vertical occipital fasciculus: a century of controversy resolved by in vivo measurements. Proc Natl Acad Sci USA 111:E5214-E5223

Yushkevich PA, Piven J, Hazlett HC, Smith RG, Ho S, Gee JC, Gerig G (2006) User-guided 3D active contour segmentation of anatomical structures: significantly improved efficiency and reliability. Neuroimage 31:1116-1128

Zhang Y, Zhang J, Oishi K, Faria AV, Jiang H, Li X, Akhter K, RosaNeto P, Pike GB, Evans A, Toga AW, Woods R, Mazziotta JC, Miller MI, van Zijl PCM, Mori S (2010) Atlas-guided tract reconstruction for automated and comprehensive examination of the white matter anatomy. Neuroimage 52:1289-1301

Zhang H, Schneider T, Wheeler-Kingshott CA, Alexander DC (2012) NODDI: practical in vivo neurite orientation dispersion and density imaging of the human brain. Neuroimage 61(4):1000-1016 